\title{
The mass function dependence on the dynamical state of dark matter haloes
}

\author{
R. Seppi ${ }^{1}$, J. Comparat ${ }^{1}$, K. Nandra ${ }^{1}$, E. Bulbul ${ }^{1}$, F. Prada ${ }^{2}$, A. Klypin ${ }^{3,4}$, A. Merloni ${ }^{1}$, \\ P. Predehl ${ }^{1}$, and J. Ider Chitham ${ }^{1}$
}

\author{
${ }^{1}$ Max-Planck-Institut für extraterrestrische Physik (MPE), Giessenbachstrasse 1, 85748 Garching bei München, Germany \\ e-mail: rseppi@mpe.mpg.de \\ 2 Instituto de Astrofísica de Andalucía (CSIC), Glorieta de la Astronomía, 18080 Granada, Spain \\ 3 Astronomy Department, New Mexico State University, Las Cruces, NM, USA \\ ${ }^{4}$ Department of Astronomy, University of Virginia, Charlottesville, VA, USA
}

Received 7 August 2020 / Accepted 25 May 2021

\begin{abstract}
Context. Galaxy clusters are luminous tracers of the most massive dark matter haloes in the Universe. To use them as a cosmological probe, a detailed description of the properties of dark matter haloes is required.

Aims. We characterize how the dynamical state of haloes impacts the dark matter halo mass function at the high-mass end (i.e., for haloes hosting clusters of galaxies).

Methods. We used the dark matter-only MultiDark suite of simulations and the high-mass objects $M>2.7 \times 10^{13} M_{\odot} h^{-1}$ therein. We measured the mean relations of concentration, offset, and spin as a function of dark matter halo mass and redshift. We investigated the distributions around the mean relations. We measured the dark matter halo mass function as a function of offset, spin, and redshift. We formulated a generalized mass function framework that accounts for the dynamical state of the dark matter haloes.

Results. We confirm the recent discovery of the concentration upturn at high masses and provide a model that predicts the concentration for different values of mass and redshift with one single equation. We model the distributions around the mean values of concentration, offset, and spin with modified Schechter functions. We find that the concentration of low-mass haloes shows a faster redshift evolution compared to high-mass haloes, especially in the high-concentration regime. We find that the offset parameter is systematically smaller at low redshift, in agreement with the relaxation of structures at recent times. The peak of its distribution shifts by a factor of $\sim 1.5$ from $z=1.4$ to $z=0$. The individual models are combined into a comprehensive mass function model, which predicts the mass function as a function of spin and offset. Our model recovers the fiducial mass function with $\sim 3 \%$ accuracy at redshift 0 and accounts for redshift evolution up to $z \sim 1.5$.

Results. This new approach accounts for the dynamical state of the halo when measuring the halo mass function. It offers a connection with dynamical selection effects in galaxy cluster observations. This is key toward precision cosmology using cluster counts as a probe.
\end{abstract}

Key words. cosmology: theory - galaxies: clusters: general - X-rays: galaxies: clusters - dark matter - cosmology: observations

\section{Introduction}

Galaxy clusters are the most massive virialized, gravitationally bound structures in the Universe. They grow hierarchically, starting from matter perturbations in the initial density field. This makes them good tracers of the underlying cosmic web and its densest regions. Clusters in cosmology are used to construct the halo mass function, which indicates the mass density of haloes in a specific volume, in a small mass interval between $M$ and $M+\mathrm{d} M$ (Weinberg et al. 2013). An early theoretical description of the mass function was given by Press \& Schechter (1974; PS) based on the assumption that Gaussian density perturbations overcoming a fixed density contrast collapse into haloes. This formally accounts for only half of the total halo mass in the Universe. An alternative approach, employing the excursion set theory, solved these shortcomings by considering the probability of crossing a given barrier with random walks (Bond et al. 1991). This provides a good prediction for high-mass haloes, but it predicts too many low-mass objects. The introduction of ellipsoidal collapse corrected these differences between simulations and theory (Sheth \& Tormen 1999, 2002).
The mass function has been extensively studied in more recent works in an attempt to find a universal model that is independent from cosmology (Jenkins et al. 2001; Tinker et al. 2008; Bhattacharya et al. 2011; Despali et al. 2016; Bocquet et al. 2016, 2020; Comparat et al. 2017). A robust way to build such a mass function model is using $N$-body simulations (Kravtsov et al. 1997; Springel 2005), for example MultiDark (Prada et al. 2012; Klypin et al. 2016). The generalization of such models as a function of cosmological parameters is best handled by emulating the mass function based on large sets of simulations (e.g., McClintock et al. 2019; Nishimichi et al. 2019; Bocquet et al. 2020). It is important to precisely predict the halo mass function to fulfill the potential of current and future X-ray, SZ, or optical cluster surveys such as eROSITA (Merloni et al. 2012; Predehl et al. 2021), Planck (Zubeldia \& Challinor 2019), SPT-3G (Benson et al. 2014), CMB S4 (Abazajian et al. 2019), SPIDERS/eBOSS (Dawson et al. 2016; Finoguenov et al. 2020), DESI (DESI Collaboration 2016), 4MOST (de Jong 2011; Finoguenov et al. 2019), Euclid (Laureijs et al. 2011), LSST (LSST Science Collaboration 2009), and WFIRST (Spergel et al. 2015). These future surveys will 

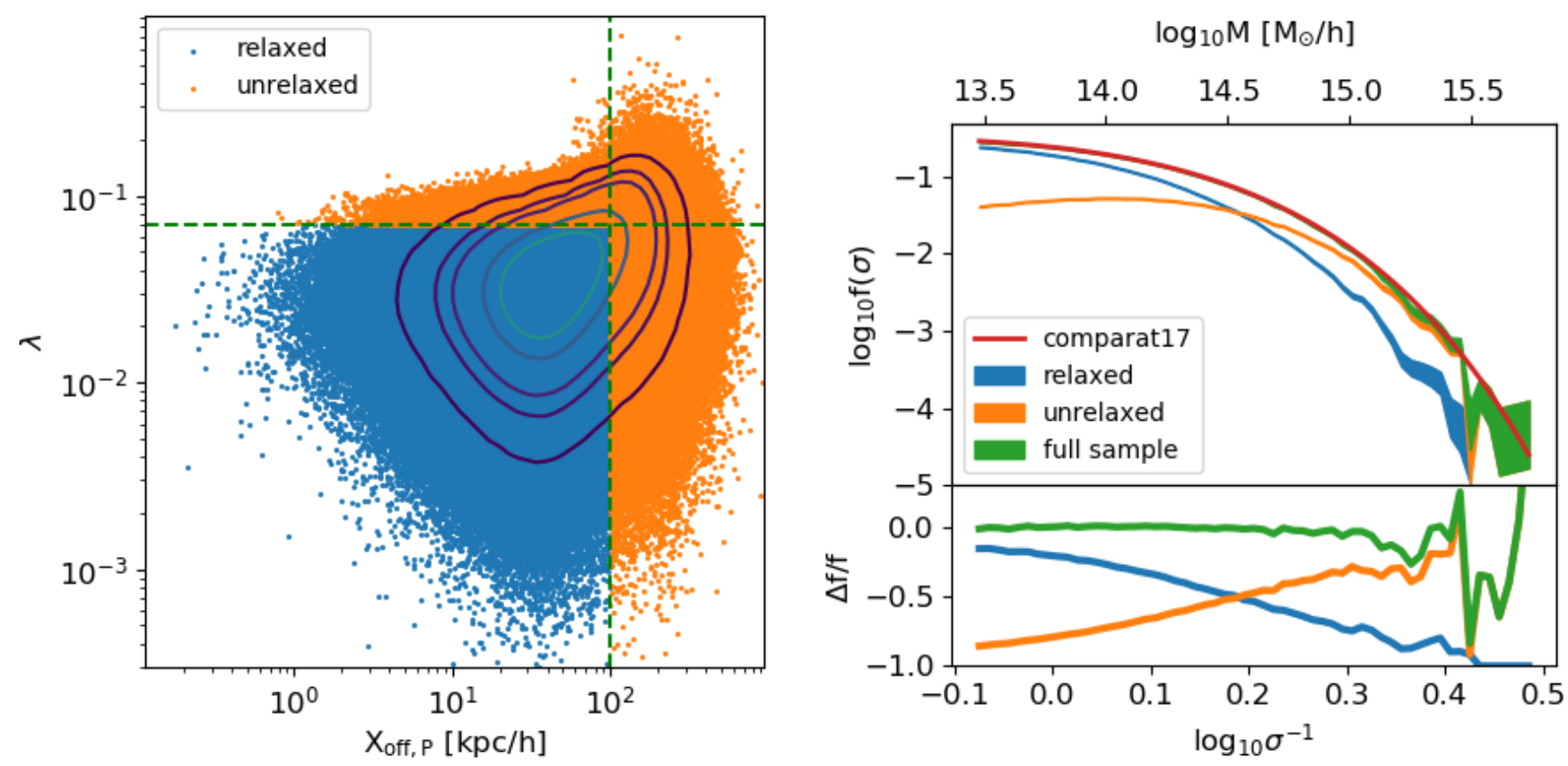

Fig. 1. Contribution of relaxed and unrelaxed haloes to the total mass function. Left panel: distribution of redshift zero dark matter haloes in the $X_{\mathrm{off}, P}$ and $\lambda$ plane. Cuts in $X_{\mathrm{off}, P}$ and $\lambda$ are applied to divide relaxed (blue) and disturbed (orange) structures. The contours contain $1 \%, 5 \%, 10 \%$, $30 \%$, and $50 \%$ of the data. Right panel: Halo mass functions vs. mass $(\sigma)$ at redshift 0 , as defined in Sect. 2 and using Eqs. (15) and (16). This mass function is built with different subsets of haloes from HMD at $z=0$ (see Sect. 3): the red line indicates the model from Comparat et al. (2017), the shaded areas represent the relaxed (blue), unrelaxed (orange), and the full sample (green) of haloes. The areas cover $1 \sigma$ uncertainties. Lower panel: residuals fraction of each component compared to the red model in the upper panel.

provide tighter constraints on mass-observable scaling relations. This means that systematic uncertainties due to the accuracy of the halo mass function model and its evolution with redshift will contribute to the total error budget significantly more than in the previous cluster counts experiments (Salvati et al. 2020). Therefore, a detailed prediction of the theoretical dark matter halo statistic is required. In this work we calibrate a mass function model that includes the dynamical properties of dark matter haloes.

Relaxed dark matter haloes can be selected according to multiple diagnostics (Neto et al. 2007; Macciò et al. 2008; Prada et al. 2012; Klypin et al. 2016): (i) the virial parameter $2 K /|W|-1$, where $K$ and $W$ are respectively the kinetic and potential energy within the virial radius; (ii) the spin parameter $\lambda=J \sqrt{E} / G M^{5 / 2}$ (Peebles 1969), which traces the dynamical state of the halo; (iii) the fraction of substructures, which is higher in unrelaxed haloes; (iv) the offset parameter $X_{\text {off }}=$ $\left|R_{\text {peak }}-R_{\text {cm }}\right| / R_{\text {vir }}$ (Behroozi et al. 2013; Klypin et al. 2016), which is the difference between the position of the peak of the density profile and the center of mass, normalized by virial radius. If the halo is perfectly relaxed, the peak of the profile will correspond to the center of mass, and $X_{\text {off }}$ will be small. On the other hand, higher $X_{\text {off }}$ values will indicate an unrelaxed halo (e.g., merger, accretion). A combination of these quantities can be used.

To illustrate the respective contribution to the mass function of relaxed and disturbed haloes, we divided the sample of haloes in HugeMultiDark (hereafter HMD, see Sect. 3) at $z=0$ using $X_{\text {off }}$ and $\lambda$. We consider the offset parameter in physical scale $X_{\mathrm{off}, P}=\left|R_{\mathrm{peak}}-R_{\mathrm{cm}}\right|$ (i.e., not normalizing by the virial radius). We consider haloes with $X_{\mathrm{off}, P}<100 \mathrm{kpc} h^{-1}$ and $\lambda<0.007$ as relaxed. In Fig. 1 we show the halo mass function of the complete halo population: the relaxed and disturbed halo population. The left panel shows the distribution of redshift zero haloes in the $X_{\mathrm{off}, P}$ and $\lambda$ plane. The right panel shows the three multiplic- ity functions (see formalism in Sect. 2) sampled by all haloes (green), relaxed haloes (blue), and disturbed haloes (orange). The bottom panel shows their relative contribution as a function of halo mass. It is clear how at $10^{13.5} M_{\odot} h^{-1}$ the contribution from relaxed structures dominates by a factor of about 0.8 dex. The multiplicity functions of the two samples cross each other at $10^{14.5} M_{\odot} h^{-1}$, then the unrelaxed structures take over at the high-mass end. This approach offers a possible connection to selection effects in observations, such as the cool core bias in X-rays (Eckert et al. 2011; Käfer et al. 2019, 2020). It possibly offers a solution to mitigate biases in a cosmological interpretation of clusters abundance. This might improve cosmological constraints using X-ray selected clusters (Ider Chitham et al. 2020).

In this article, we investigate the variations of the dark matter halo mass function as a function of the dynamical state of the constituting haloes. To trace the dynamical state we use $X_{\text {off }}$ and $\lambda$. This paper is structured as follows. We define the formalism in Sect. 2. We present the $N$-body data used in Sect. 3. We present the average relations between concentration, $X_{\text {off }}$, spin, and mass, as well as their distribution around mean values, in Sect. 4. We define the generalized mass function framework in Sect. 5. We present the generalized model of the halo mass function as a function of $X_{\text {off }}$ and $\lambda$ in Sect. 6. We present the results of the fit and the best-fit parameters in Sect. 7. We summarize our findings in Sect. 8, and discuss their implications for cosmological studies.

\section{Formalism and definitions}

The growth of the density perturbations in the matter field is described by the evolution of the overdensity field and its variance as a function of scale. The variance of the smoothed density 
R. Seppi et al.: The mass function dependence on the dynamical state of dark matter haloes

Table 1. Correspondence between mass, peak height, and variance of the linear density field at $z=0$ and $z=0.5$.

\begin{tabular}{|c|c|c|c|c|}
\hline \multirow{2}{*}{$\begin{array}{l}\text { Mass } \\
\log _{10} \\
M_{\odot} h^{-1}\end{array}$} & \multicolumn{2}{|c|}{$z=0$} & \multicolumn{2}{|c|}{$z=0.5$} \\
\hline & $v=\delta_{c} / \sigma$ & $\log _{10}(1 / \sigma)$ & $v=\delta_{c} / \sigma$ & $\log _{10}(1 / \sigma)$ \\
\hline 10 & 0.446 & -0.577 & 0.579 & -0.465 \\
\hline 10.5 & 0.506 & -0.523 & 0.657 & -0.41 \\
\hline 11 & 0.58 & -0.463 & 0.752 & -0.35 \\
\hline 11.5 & 0.673 & -0.399 & 0.872 & -0.286 \\
\hline 12 & 0.79 & -0.329 & 1.02 & -0.216 \\
\hline 12.5 & 0.942 & -0.253 & 1.22 & -0.14 \\
\hline 13 & 1.14 & -0.17 & 1.48 & -0.0568 \\
\hline 13.5 & 1.41 & -0.0779 & 1.83 & 0.035 \\
\hline 14 & 1.78 & 0.0236 & 2.31 & 0.137 \\
\hline 14.5 & 2.31 & 0.137 & 3 & 0.25 \\
\hline 15 & 3.09 & 0.263 & 4.01 & 0.376 \\
\hline 15.5 & 4.29 & 0.405 & 5.56 & 0.518 \\
\hline
\end{tabular}

Notes. These quantities are described by Eqs. (1) and (2).

field is defined as

$\sigma^{2}(M, z)=\int \frac{\mathrm{d}^{3} k}{(2 \pi)^{3}} \hat{W}^{2}(k, R) P(k, z)$,

where $k$ is the wavenumber, $\hat{W}$ the Fourier transform of a top-hat filtering function, and $P(k, z)=D_{+}(z)^{2} P(k, 0)$ the linear matter power spectrum. Its redshift evolution is encoded in the growth factor $D_{+}(z)$. The quantity

$v(M, z)=\delta_{\mathrm{cr}}(z) / \sigma(M, z)$

is the peak height, where $\delta_{\text {cr }}$ is the critical overdensity required for a structure to collapse in a dark matter halo (Press \& Schechter 1974; Sheth \& Tormen 2002). We report explicit values of mass, $\sigma$, and $v$ in Table 1 for $z=0$ and $z=0.5$.

We write the mass function in its differential form as in Eq. (3) (see Allen et al. 2011, for a recent review),

$\frac{\mathrm{d} n}{\mathrm{~d} \ln M}=\frac{\rho_{m}}{M}\left|\frac{\mathrm{d} \ln \sigma}{\mathrm{d} \ln M}\right| f(\sigma)$

where $f(\sigma)$ is the multiplicity function. A comprehensive list of models of the multiplicity function is available in Table 1 of Murray et al. (2013).

In this paper as mass variable we use $\sigma$ (Eq. (1)), peak height (Eq. (2)), and mass interchangeably.

\section{Simulations}

We describe the set of gravity-only simulations and the halo finding post-process in this section.

\subsection{MultiDark}

We use the MultiDark simulations (Prada et al. 2012; Riebe et al. 2013; Klypin et al. 2016). They are computed in a flat $\Lambda$ CDM Planck (Planck Collaboration XI 2014) cosmology $\left(H_{0}=67.77 \mathrm{~km} \mathrm{~s}^{-1} \mathrm{Mpc}^{-1}, \Omega_{m 0}=0.307115, \Omega_{b 0}=0.048206\right.$, $\sigma_{8}=0.8228$ ) with the GADGET-2 code (Springel 2005). It is one of the largest sets of high-resolution $\left(\sim 4000^{3}\right.$ particles $) \mathrm{N}$-body simulations.
Table 2. $N$-body simulations used in this analysis.

\begin{tabular}{lcccc}
\hline \hline Name & $L$ & $M_{\mathrm{p}}$ & $M_{\min }$ & $N$ haloes \\
\hline HMD & 4.0 & $7.9 \times 10^{10}$ & $2 \times 10^{13}$ & 13330574 \\
BIGMD & 2.5 & $2.4 \times 10^{10}$ & $5 \times 10^{12}$ & 27575832 \\
MDPL2 & 1.0 & $1.51 \times 10^{9}$ & $4 \times 10^{11}$ & 17036888 \\
\hline
\end{tabular}

Notes. $L$ : length of the box in Gpc $h^{-1} . M_{p}$ : mass of the particle in $M_{\odot} h^{-1} \cdot M_{\min }:$ minimum halo mass considered $M_{\mathrm{vir}}>M_{\min }$ in $M_{\odot} h^{-1}$. Number of haloes in the snapshots at $z=0$.

We used three MultiDark simulations: HMD, BIGMD, MDPL2 (see details in Table 2). Alternative simulations that could be used for this project include MILLENNIUM-XXL, DARKSKIES, Q CONTINUUM, $V^{2}$ GC SIMULATION, described in Angulo et al. (2012), Skillman et al. (2014), Heitmann et al. (2015), and Ishiyama et al. (2015) respectively.

The complete list of simulation outputs (snapshots) utilized are given in Table A.6, where the expansion parameter $a$ and the corresponding redshifts are reported for each snapshot.

\subsection{Halo finding}

Finding haloes in dark matter simulations is not an easy task (see Knebe et al. 2013; Behroozi et al. 2015, for a review). In this study haloes are identified by the Robust Overdensity Calculation using K-Space Topologically Adaptive Refinement (ROCKSTAR) and CONSISTENTTREES algorithms (Behroozi et al. 2013). They are based on adaptive hierarchical refinement of friends-of-friends (FOF) groups. They work with six phasespace dimensions (halo positions and velocities), and one time dimension. This allows us to track relative motions and merging history between substructures in different snapshots. ROCKSTAR computes the halo mass of identified objects by removing the unbound particles inside the virial radius. Virial mass and virial radius are related by

$M_{\mathrm{vir}}(z)=\frac{4}{3} \pi \Delta_{\mathrm{vir}}(z) \Omega_{\mathrm{M}}(z) \rho_{\mathrm{b}}(z) R_{\mathrm{vir}}^{3}$,

where $R_{\text {vir }}$ encompasses a mean halo density equal to the background matter density multiplied by $\Delta_{\mathrm{vir}}, \Omega_{\mathrm{M}}$ is the matter density parameter, and $\rho_{\mathrm{b}}$ is the matter density of the Universe. The overdensity over the matter background $\Delta_{\text {vir }}$ is defined according to Bryan \& Norman (1998) as

$$
\begin{aligned}
\Delta_{\text {vir }} & =\left(18 \pi^{2}+82 x-39 x^{2}\right) / \Omega(z), \\
\Omega(z) & =\Omega_{M, 0}(1+z)^{3} / E(z)^{2},
\end{aligned}
$$

where $x=\Omega(z)-1, \Omega_{M, 0}$ is the matter density parameter at the present day, and $E(z)$ is the Hubble parameter as a function of redshift in units of $H_{0}$ :

$E(z)=\frac{H(z)}{H_{0}}=\sqrt{\Omega_{0, \mathrm{M}}(1+z)^{3}+\Omega_{\Lambda}}$.

Given the reference cosmology adopted in this work, the virial overdensity is equal to 332.5 at $z=0$ and asymptotically tends to 178 at high redshift.

The recovery of main properties such as position, mass, and circular velocity is consistent between different finders. However, derived properties such as spin show a $20 \%$ scatter (Knebe et al. 2013). This holds especially for low-mass haloes 
with fewer than 30-40 particles, where the identification of substructures is not straightforward (Knebe et al. 2011). The lowmass limits in Table 2 are set at more than $\sim 200$ particles per halo in all boxes. This ensures accurate halo properties (Behroozi et al. 2013; Knebe et al. 2013).

In this work we use the virial overdensity (Eq. (5)). The virial mass function has been shown to be the one that comes closest to universality (Despali et al. 2016).

\section{Concentration, offset, spin: Empirical relations with peak height and redshift}

In the footsteps of Klypin et al. (2016) and Rodriguez-Puebla et al. (2016), we analyze the average relations linking concentration, $\lambda$, and $X_{\text {off }}$ to the peak height, respectively in Sects. 4.14.3. We also analyze the distributions of these quantities around the mean relations. The mean relations are fitted by models that simultaneously account for the mass and redshift dependence of the relations (Eqs. (9), (11), and (13)). The probability density functions (PDFs) of concentration, spin, and $X_{\text {off }}$ are fitted by modified Schechter models, respectively in Eqs. (10), (12), and (14). The PDFs at different redshifts are modeled independently.

\subsection{Concentration-mass-redshift relation}

We study the relation between concentration and mass. Numerical simulations (Navarro et al. 1996) show that to a good approximation $(\sim 10-20 \%)$, the density of dark matter haloes is described by the profile in Eq. (6),

$\rho(r)=\frac{\rho_{s}}{\left(r / R_{s}\right)\left(1+r / R_{s}\right)^{2}}$,

where $R_{s}$ is the scale radius. The characteristic density of the halo $\rho_{s}$ is equal to

$\rho_{s}=\rho_{\text {crit }} \frac{\Delta}{3} \frac{c^{3}}{\ln (1+c)-c /(1+c)}$,

where $c$ is the concentration, $\Delta$ the overdensity, and $\rho_{\text {crit }}$ is the critical density of the Universe. The concentration is a dimensionless quantity defined by

$c_{\Delta}=R_{\Delta} / R_{s}$

where $\Delta$ can refer to any threshold. In this work we consider the virial overdensity over the matter background $\Delta_{\text {vir }}$ (see Eq. (5)). Therefore, we study the virial concentration $c_{\mathrm{vir}}=R_{\mathrm{vir}} / R_{\mathrm{s}}$. The concentration-mass relation is an important part of models describing galaxy clusters (e.g., reviews from Allen et al. 2011; Umetsu 2020) or gravitational lensing (e.g., reviews from Bartelmann 2010; Kilbinger 2015).

This relation has been extensively studied in simulations. The concentration anti-correlates with mass with negative redshift trend (e.g., Diemer \& Joyce 2019; Ragagnin et al. 2019). Its detailed trend depends on the measurement method, in simulations (Meneghetti \& Rasia 2013; Lang et al. 2015; Poveda-Ruiz et al. 2016) and in observations (Foëx et al. 2014; Phriksee et al. 2020; Du et al. 2015; Shan et al. 2017). This introduces possibles biases in the measure of concentration (Sereno et al. 2015; Cibirka et al. 2017; van Uitert et al. 2016). Diemer \& Kravtsov (2015) found that the relation shows the smaller deviation from universality when adopting the definition $c_{200 c}$. However, it is not completely universal, meaning that concentration is described not only by mass or $v$, but also by assembly history. Leaving aside biases in definition and measurement, different models have been proposed to describe it: power laws (e.g., Duffy et al. 2008; Dutton \& Macciò 2014); a combination of power laws to describe the high-mass upturn (e.g., Klypin et al. 2016; Diemer \& Joyce 2019); semi-analytic models based on the Press-Schechter theory (Correa et al. 2015). In this section we extend the models from Klypin et al. (2016). We adjust a global model that includes a redshift dependence for high-mass haloes at relatively low redshift $(z<1.5)$, which is particularly interesting for haloes hosting galaxy clusters.

\subsubsection{Model}

We model the concentration $c$ as a function of the rms of the overdensity field $\sigma(M, z)$ (not as the function of halo mass $M$ ). Our parameterization of the concentration- $\sigma$ relation is a generalization of the Klypin et al. (2016) relation and reads

$$
\begin{aligned}
c(\sigma, z)= & \frac{b_{0}}{(1+z)^{0.2}}\left[1+7.37\left(\frac{\sigma}{a_{0}(1+z)^{1 / 2}}\right)^{3 / 4}\right] \ldots \\
& {\left[1+0.14\left(\frac{\sigma}{a_{0}(1+z)^{1 / 2}}\right)^{-2}\right] . }
\end{aligned}
$$

The best-fit values are in Table A.1. We find best-fit values of $a_{0}=0.754091 \pm 0.000004, b_{0}=0.574413 \pm 0.000002$, in agreement with Klypin et al. (2016). This model is fitted using haloes with $M>10^{12.5} M_{\odot} h^{-1}(v \sim 0.95$ at $z=0)$. The lowmass end is not sampled due to the particle mass resolution. We do not consider a high-redshift regime where there is a statistical limitation of high-mass haloes. We chose the binning limits following Klypin et al. (2016). The strength of our model resides in the ability to predict the concentration $-\sigma$ relation for a variety of masses and redshifts with a single equation. We recover the hockey stick shape of the relation (see Fig. 2). This is consistent with Prada et al. (2012), Klypin et al. (2016), and Diemer \& Joyce (2019) (the last also includes a cosmology dependence). This upturn feature is absent in Duffy et al. (2008) and Wang et al. (2020), due to the smaller volumes analyzed (400 Mpc $h^{-1}$ and $500 \mathrm{Mpc}^{-1}$, respectively). They are not large enough to obtain a significant number of high-mass objects and probe the upturn.

We describe the distribution of concentration around its mean value and we model its probability density function. We use snapshots from HMD, at four redshifts. Each snapshot is divided into six slices of mass. The PDFs obtained at each redshift are fitted simultaneously. It includes a $\sigma$ dependence in our model (see Eq. (10)):

$P(c, \sigma)=A\left(\frac{c}{x_{0} \sigma^{e_{0}}}\right)^{\alpha \sigma^{e_{1}}} \exp \left[-\left(\frac{c}{x_{0} \sigma^{e_{0}}}\right)^{\beta \sigma^{e_{2}}}\right]$.

The best-fit parameters are included in Table A.1. Uncertainties are negligible for most parameters. The distribution of concentration around its mean value is well described by a modified Schechter function with mass-dependent terms (Eq. (10)). We use the best-fit model to predict the distribution of concentration in fixed mass slices as a function of redshift. The result is shown in Fig. 3. The full distribution of concentration (not sliced in different mass intervals) is well described by a modified Schechter as well. 


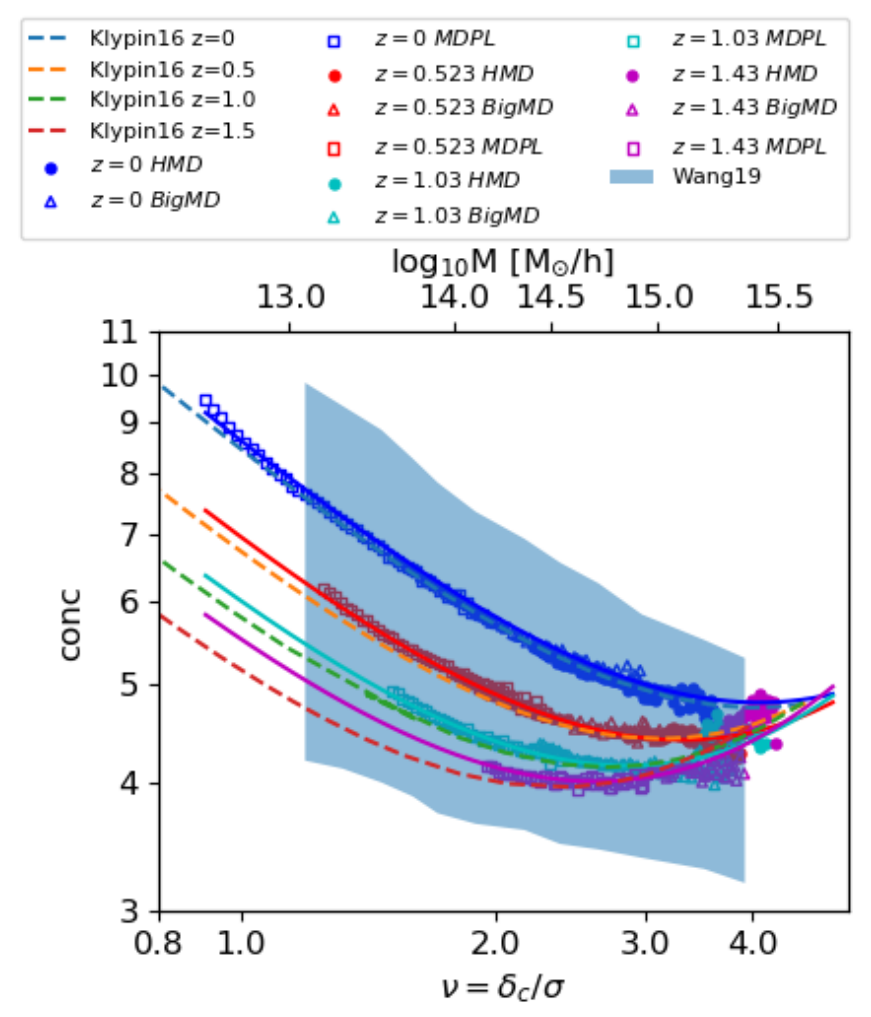

Fig. 2. Concentration- $\sigma$ relation (Eq. (9)). Circular dots, triangles, and squares represent HMD, BIGMD, MDPL2, respectively. They are colorcoded by redshift. Straight lines indicate our best-fit model, dotted lines show the model from Klypin et al. (2016), and the shaded blue area indicates the distribution from Wang et al. (2020) at $z=0$. The upper $x$-axis converts peaks values into mass at $z=0$.

\subsubsection{Discussion}

The average value of concentration increases at late times. It goes from 4 at $z=1.43$ to 5.8 at $z=0$ for haloes corresponding to peak height $v=2$. We confirm the recent discovery of the concentration upturn at high masses (Klypin et al. 2016; Diemer \& Joyce 2019). Klypin et al. (2016) suggest that the high-mass upturn is caused by the tendency of regions with smaller root mean square variance in the overdensity field to be more spherical. These regions are the ones that evolve into high-mass dark matter haloes. Because of this aspect, gravitational accretion of matter toward the center is more efficient and results in higher concentration. In addition, very massive haloes correspond to the highest peaks of the density field. This means that the probability of finding them in a phase characterized by strong accretion and therefore higher concentration is higher, as shown by Ludlow et al. (2012).

We find that the concentration of haloes with different masses shows a different evolution with redshift (see Fig. 3). At $z=0$ the concentration of high-mass haloes gives a smaller contribution to the total PDF in the high-concentration tail. At higher redshift we see high-concentration haloes with both high and low mass. The opposite holds for low-mass haloes, which contribute more to the total PDF at higher $z$ in the low-concentration regime. At high redshift the distribution of concentration for different mass bins has the same shape. Conversely, at low redshift this statement does not hold. The low-mass distribution is much broader than the high-mass one. There is also a general redshift trend: with decreasing redshift the number of highconcentration, low-mass haloes increases. More recently in time the distribution is flatter; in fact, the slope of the power-law is smaller, going from $\sim 4.5$ at $z=1.43$ to $\sim 1.4$ in the present day. Moreover, the mass trend of the exponential decay at $z=0$ is negative with sigma $\left(e_{2}=-0.959\right)$, which translates into a faster decrease at high mass. This means that the distribution of concentration of low-mass haloes evolves more than that of highmass objects. For example, at concentration $c=8$ the PDF of high-mass haloes $\left(1 \times 10^{14}<M<2 \times 10^{14} M_{\odot} h^{-1}\right)$ changes by 0.32 dex between $z=1.43$ and $z=0$, while for low-mass objects $\left(2 \times 10^{13}<M<3 \times 10^{13} M_{\odot} h^{-1}\right)$ it varies by 0.49 dex. There is a net difference of 0.17 dex between the two, meaning that the high end of concentration evolves $\sim 1.5$ times faster for lower mass haloes compared to high-mass ones. This is in agreement with the fact that these haloes evolve in different environments. This causes a different redshift evolution of the distribution of concentration of haloes with different masses. Structures with the same mass but different formation histories present different properties. This aspect is related to the notion of assembly bias (Gao et al. 2005; Croton et al. 2007; Angulo et al. 2008). There is a correlation between halo concentration and the age of the universe when the progenitor reached a fixed fraction of its current mass (Zhao et al. 2009). Moreover, a faster mass accretion causes a slower increase of concentration. Very massive objects reside in highly populated environments, which slows down the evolution of concentration (Zhao et al. 2003). This can be used to predict concentration as a function of assembly history (Giocoli et al. 2012; Ludlow et al. 2013, 2014). The latter is also related to the evolution of cosmological parameters. This allows modeling of the concentration mass relation for cold and warm dark matter haloes (Ludlow et al. 2016). In general, high-mass haloes cluster more than low-mass ones. Low-mass objects, some of which are found in isolated environments, will experience different histories. Isolated low-mass haloes will be highly concentrated, while the concentration of low-mass haloes in dense environments will stay low. Contreras et al. (2019) found that low-concentration haloes cluster more than haloes with high concentration at high redshift. This is in agreement with our results.

\subsection{Spin-mass-redshift relation}

In this subsection we study the relation between the spin parameter and mass. An accurate theoretical description of the distribution of the halo spin is key to understanding the possible implications of the systemic rotation on cluster sample definitions. This rotation is induced by a combination of the initial spin of the halo, the infalling material, and the merging activity.

Measurements of cluster rotation were obtained at low redshift, using member galaxies to infer the rotation movement (Hwang \& Lee 2007; Tovmassian 2015; Manolopoulou \& Plionis 2017; Bilton et al. 2019). The spin in galaxy clusters has also been studied in X-rays (Bianconi et al. 2013; Eckert et al. 2019). Highresolution data are needed to explore this topic, in the optical (Song et al. 2018) and X-ray (Hitomi Collaboration 2018) bands. Moreover, cluster cores can be analyzed by distortion of the $6.7 \mathrm{keV}$ line (Sunyaev et al. 2003), fluctuations in the CMB due to rotation (Rephaeli 1995; Cooray \& Chen 2002), and rotational kSZ effect (Baxter et al. 2019). Inferring motion with the SZ effect was also demonstrated for relaxed clusters with a significant spin on simulated (hydrodynamically) clusters (Baldi et al. 2018, 2019). Future SZ and X-rays surveys might enable such measurements on a large number of clusters. A statistical description of the halo population as a function of spin is thus of interest, and is developed in this section. 


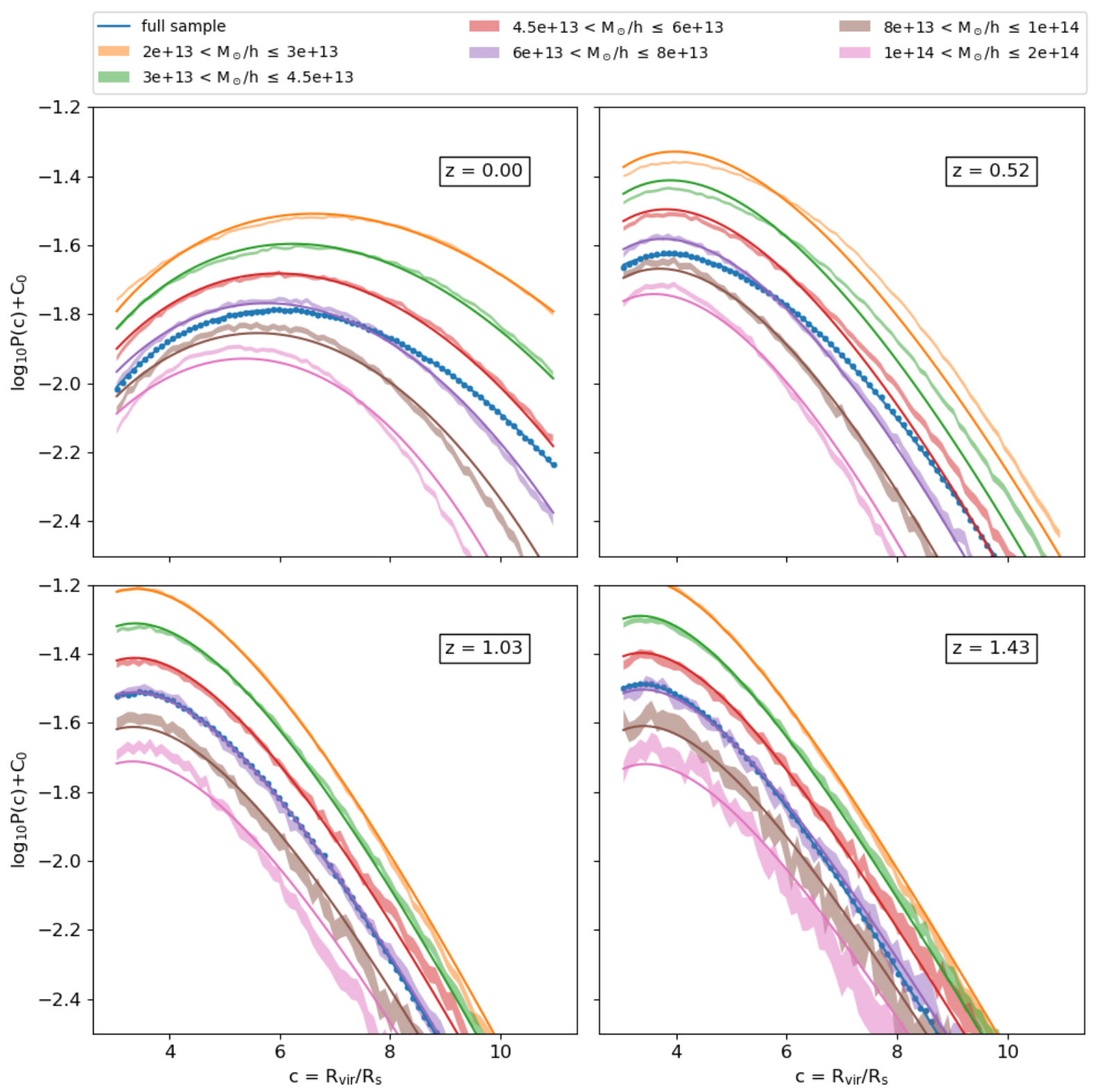

Fig. 3. Probability density function of the concentration (Eq. (10)) at the different redshifts values given in each panel. Each set is divided into mass slices, and is color-coded accordingly. The shaded areas represent the data with $1 \sigma$ error, while the straight lines indicate the best-fit model. The blue points and line represent the total sample not sliced in mass. For clarity, each line and its fit is shifted by 0.1 dex on the $y$-axis. This means that the constant $C_{0}$ assumes values of $(+0.3,+0.2,+0.1,0.0,-0.1,-0.2)$. The purple line is not shifted, and is thus the one with the proper normalization.

\subsubsection{Model}

We analyze the spin-mass-redshift relation and its PDF. The spin is defined as $\lambda=J E^{1 / 2} / G M^{5 / 2}$ (Peebles 1969). We fit the mean relation with mass as a linear relation (Eq. (11)).

$\lambda(\sigma)=a_{0}+b_{0} \sigma$.

There is no noticeable dependence on redshift, so we do not consider a redshift evolution in this model. The best-fit parameters are reported in Table A.2. The relation is shown in Fig. 4. We find that the spin correlates weakly with halo mass, as Bett et al. (2007) and Rodriguez-Puebla et al. (2016) did. The PDF of the spin parameter is best fit by a modified Schechter law with no mass dependence:

$P(\lambda)=A\left(\frac{\lambda}{x_{0}}\right)^{\alpha} \exp \left[-\left(\frac{\lambda}{x_{0}}\right)^{\beta}\right]$.
Distributions for different redshift snapshots are shown in Fig. 5 (see Table A.2 for best-fit parameters). The result is consistent with previous findings, (e.g., Rodriguez-Puebla et al. 2016). The evolution with redshift of the PDF shows that with time haloes build up higher spins: the maximum of the PDF shifts to higher spin values when redshift decreases (Fig. 5). We also tried a lognormal distribution as a model, but it was not successful.

\subsubsection{Discussion}

The small correlation with mass and the well-modeled evolution with redshift makes it a rather simple dependence to account for in statistical studies of the halo population. From the perspective of the measurement of the halo mass function based on a cluster sample, marginalizing over the spin is possible, with 


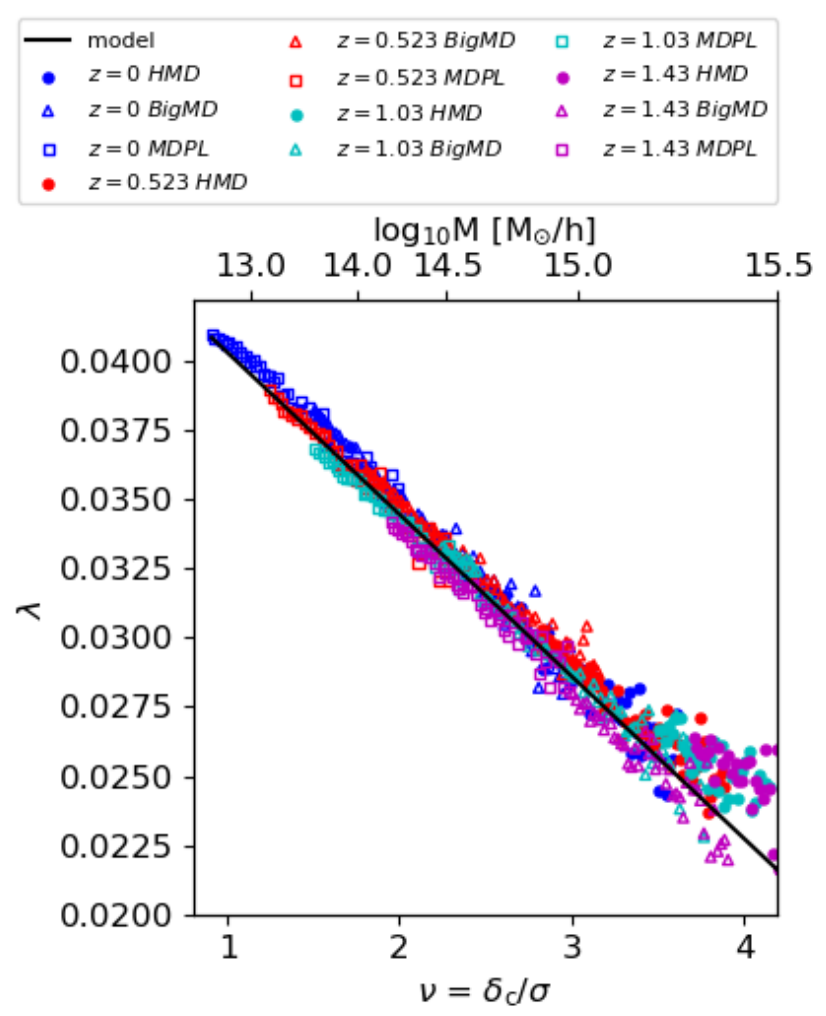

Fig. 4. Spin-mass relation $(\lambda-\sigma$, Eq. (11)). The model is a linear relation with no redshift trend. Data points are color-coded by redshift, while the different geometrical shapes refer to different simulations: squares for HMD, triangles for BigMD, and circles for MDPL. The straight line indicates the best-fit model, which considers all simulations and redshift at the same time. The best-fit parameters are given in Table A.2. The upper $x$-axis converts peaks values into mass at $z=0$.

limited complications. It also shows the spin cannot be considered a candidate for assembly bias.

\subsection{Offset-mass-redshift relation}

The offset parameter $X_{\text {off }}$ traces the relaxation state of the halo (Thomas et al. 2001; Neto et al. 2007; Henson et al. 2017). Hollowood et al. (2019) analyzed the miscentering in SDSS galaxies followed up with Chandra X-ray observations. Provided there is a link between $X_{\text {off }}$ and the brightest cluster galaxy (BCG) to $X$-ray displacement, an estimation of the bi-variate mass and $X_{\text {off }}$ function from observations of clusters is possible. To interpret it requires a detailed description of the link between $X_{\text {off }}$ and mass, which is detailed in this section. We find that lowmass haloes have smaller offset than high-mass ones at each redshift. Moreover, the offset parameter is reduced by a factor of $\sim 1.5$ from $z \sim 1.5$ to $z=0$. This is in agreement with the fact that structures relax in time.

\subsubsection{Model}

We model the $X_{\text {off }}-\sigma$ relation with a redshift dependent power law, see Eq. (13),

$\log _{10} X_{\mathrm{off}}=\frac{a_{0}}{E(z)^{0.136}} \sigma^{b_{0} E(z)^{-1.11}}$

where $E(z)$ is the dimensionless Hubble parameter. The best-fit parameters are given in Table A.3. We find $a_{0}=-1.30418 \pm$

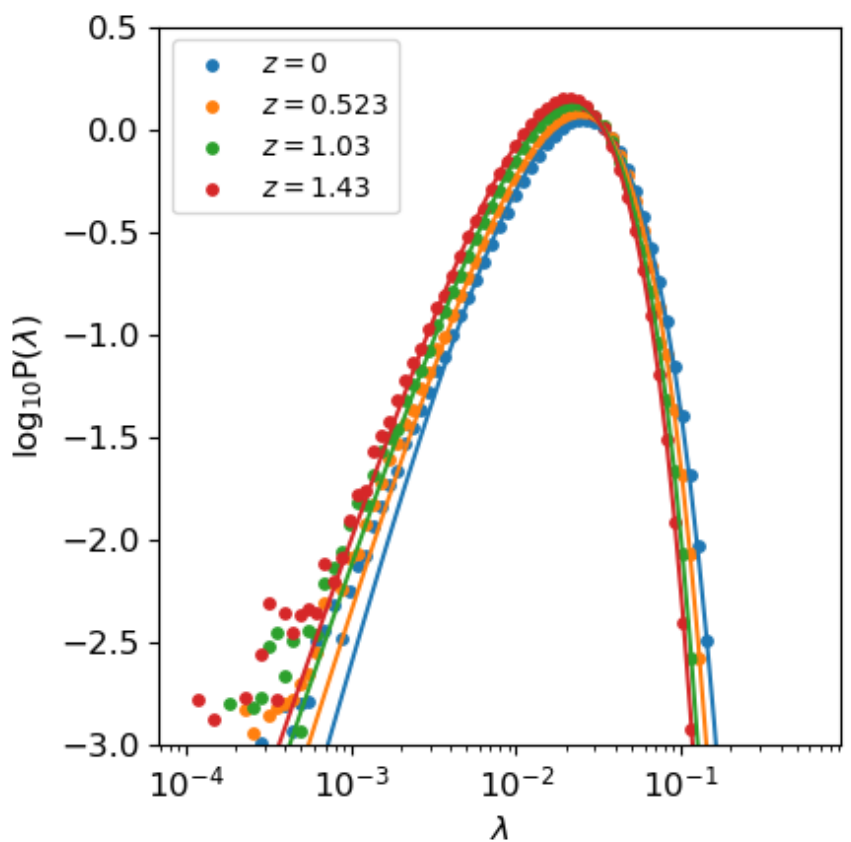

Fig. 5. PDF of $\lambda$ (Eq. (12)). Individual points represent spin bins used to compute the distribution; straight lines refer to the best-fit modified Schechter model. They are color-coded by redshift. We do not consider mass dependence in this relation. Each redshift slice is fitted independently. The best-fit parameters are given in Table A.2.

$0.00001, b_{0}=0.15084 \pm 0.00001$. We find a significant redshift evolution of the normalization and the slope of the relation. The data obtained from MultiDark simulations and the best-fit models are shown in Fig. 6. The best-fit parameters of the $X_{\text {off }}-\sigma$ relation and the distribution of $X_{\text {off }}$ are given in Table A.3. As for concentration, the strength of this equation relies upon its ability to predict an average $X_{\text {off }}$ value given the mass and the redshift of a dark matter halo.

We describe the distribution of $X_{\mathrm{off}}$ around its mean value with a modified Schechter function (Eq. (14)). The PDF of $X_{\text {off }}$ does not show mass dependency. It is included in the normalization to the virial radius $R_{\mathrm{vir}} \alpha M_{\mathrm{vir}}^{1 / 3}$. Therefore, we do not consider any $\sigma$ dependence:

$P\left(X_{\mathrm{off}}\right)=A\left(\frac{X_{\mathrm{off}}}{x_{0}}\right)^{\alpha} \exp \left[-\left(\frac{X_{\mathrm{off}}}{x_{0}}\right)^{\beta}\right]$.

We fit all haloes in each redshift snapshot together. Figure 7 shows distributions at different redshifts, fitted independently from one another. The parameters are given in Table A.3.

\subsubsection{Discussion}

We find a non-zero slope for the relation between $X_{\text {off }}$ and mass. On average, high-mass haloes have a larger offset parameter. This is described by the negative $a_{0}$ parameter. This is in agreement with the hierarchical picture of structure formation. Highmass haloes formed recently and have not had time to dynamically relax. A further contribution is given by the environment surrounding these structures. High-mass haloes form in the knots of the large-scale structure where more matter is available for inflows and mergers, making these objects more disturbed. In a picture where $X_{\text {off }}$ possibly traces the cool core-non-cool core classification of galaxy clusters in X-rays (Eckert et al. 2011), massive structures have a higher fraction of non-cool cores. This 

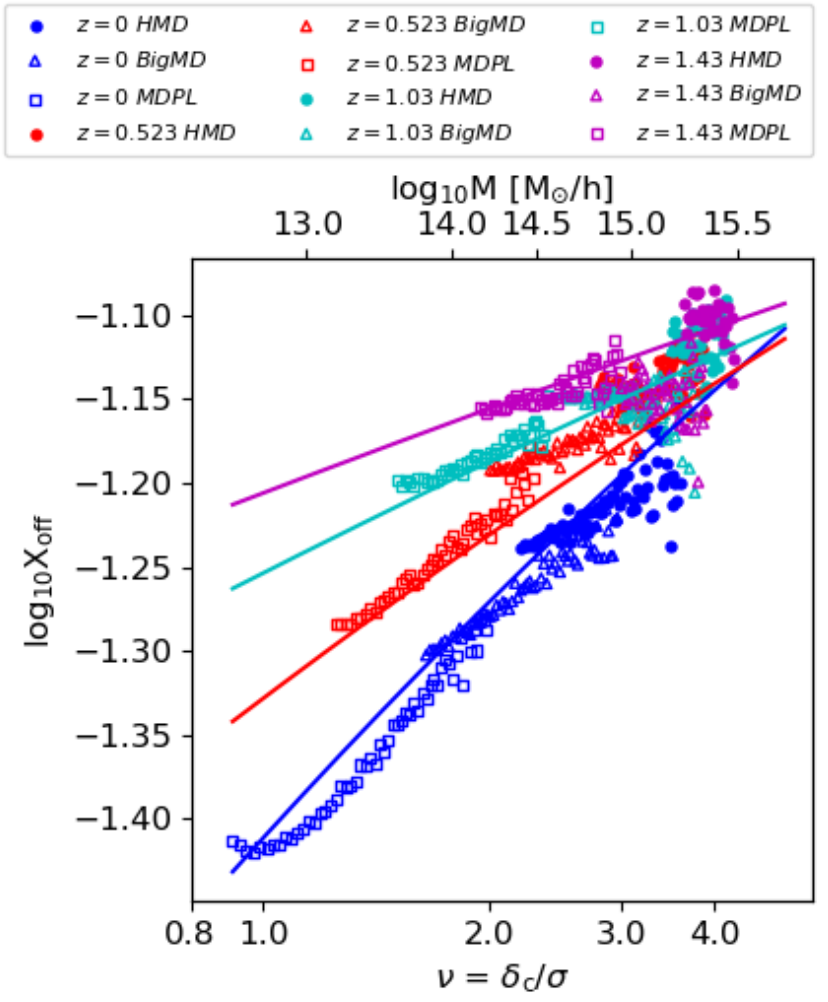

Fig. 6. Offset-mass relation $\left(X_{\text {off }}-\sigma\right.$, Eq. (13)). Circular dots, triangles, and squares represent HMD, BIGMD, and MDPL2 respectively. They are color-coded by redshift. Straight lines show the best-fit model. The bestfit parameters are given in Table A.3. The upper $x$-axis converts peaks values into mass at $z=0$.

influences the high-mass tail of the mass function (see Fig. 1). Therefore, given an X-ray flux limit, the mass function of a relaxed galaxy clusters sample will be complete to lower masses than an unrelaxed one. This effect also evolves with redshift. At high $z$, haloes show a larger offset. In this context it means that it is more difficult to detect structures at earlier times. More recently, structures have had time to relax and therefore show smaller values of the offset parameter. This is linked to the development of cool cores at low redshift (Ettori \& Brighenti 2008). The PDF shows a power-law growth from low offset values (slope $\alpha=3.71$ at $z=0$ ) and exponential cutoff at high $X_{\text {off }}$. It is described by a modified Schechter function (Eq. (14)). Its maximum shifts by a factor of $\sim 1.5$, from $X_{\text {off }} \sim 0.09$ to $X_{\text {off }} \sim 0.06$, between redshift 1.4 and 0 , confirming that haloes have more time to relax. The shape of the distribution does not show a significant redshift trend. The width of the probability density functions, measured at $\log _{10} P\left(X_{\text {off }}\right)=-2.5$, at $z=0$ and $z=1.4$ agree with $2.8 \%$ accuracy. At $z=0$ these values span from $X_{\text {off }} \sim 0.01$ to $X_{\text {off }} \sim 0.3$. This precludes the offset from being a possible assembly bias candidate.

\section{Generalized mass function}

Generalizations of the mass function have been made in a number of directions: cosmological parameters, angular momentum, and friction (Achitouv \& Corasaniti 2012; Achitouv et al. 2014; Del Popolo et al. 2017) via detailed modeling of the collapse barrier. Nevertheless, it is technically demanding to connect these parameters to observations. In this section we generalize the mass function formalism to include additional variables $\left(X_{\mathrm{off}}, \lambda\right)$

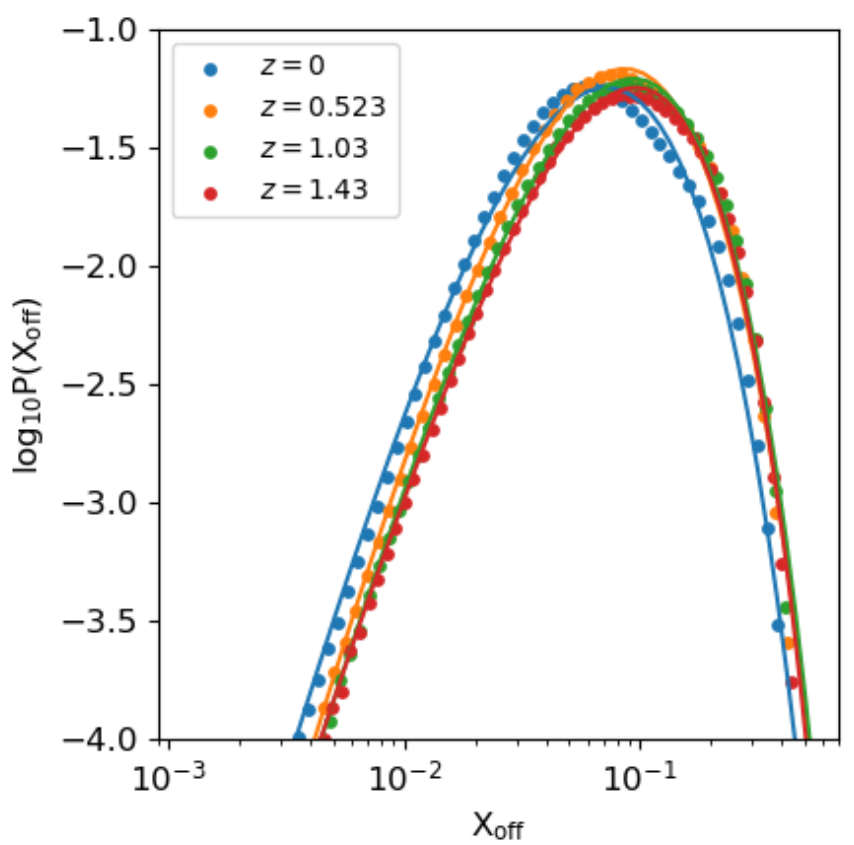

Fig. 7. Probability density function of $X_{\text {off }}$. Each panel shows the distribution at a specific redshift. Scatter points indicate the data, while straight lines represent the modified Schechter model. The samples are color-coded by redshift. Each redshift slice is fitted independently by Eq. (14). The best-fit parameters are given in Table A.3.

in its formulation. These two quantities describe the properties of dark matter haloes and will hopefully become connectable to observational properties.

\subsection{Definitions}

We compute the mass function as follows:

$\frac{\mathrm{d} n}{\mathrm{~d} \ln M}=\frac{\Delta N_{M}}{V \Delta \ln M}$

Here $\Delta N_{M}$ is the number of haloes in each mass bin $\Delta \ln M$ and $V$ is the total volume of the simulation. It corresponds to Eq. (3). The $M(\sigma)$ relation and its first derivative are computed with Colossus (Diemer 2018). By convention, the relation between a certain mass and its corresponding scale is normalized with the matter density at $z=0$. By combining the previous equations with Eq. (3), we estimate a multiplicity function:

$f(\sigma)=\frac{\mathrm{d} n}{\mathrm{~d} \ln M} \frac{M}{\rho_{m}}\left(\frac{\mathrm{d} \ln \sigma^{-1}}{\mathrm{~d} \ln M}\right)^{-1}$.

\subsection{Generalization}

We include the offset parameter and spin by generalizing the approach described in the previous section. The number density of haloes as a function of mass and dynamical state is described by Eq. (17):

$\frac{\mathrm{d} n}{\mathrm{~d} \ln M \mathrm{~d} \log X_{\mathrm{off}} \mathrm{d} \log \lambda}=\frac{\Delta N_{M, X_{\mathrm{off}}, \lambda}}{V s_{M} s_{X_{\mathrm{off}}} s_{\lambda}}$.

Here $\Delta N_{M, X_{\mathrm{off}}, \lambda}$ is the number of haloes in each mass, $X_{\text {off }}$ and $\lambda$ bin, $V$ is the total volume of the simulated cube and $s_{M}, s_{X_{\text {off }}}$, and 
$s_{\lambda}$ are the natural (base 10) logarithm of mass $\left(X_{\mathrm{off}}, \lambda\right)$ binning. Equivalently to Eq. (16), we calculate

$h\left(\sigma, X_{\mathrm{off}}, \lambda\right)=\frac{\mathrm{d} n}{\mathrm{~d} \ln M \mathrm{~d} \log X_{\text {off }} \mathrm{d} \log \lambda} \frac{M}{\rho_{m}}\left(\frac{\mathrm{d} \ln \sigma^{-1}}{\mathrm{~d} \ln M}\right)^{-1}$.

We consider a single integration of $h\left(\sigma, X_{\mathrm{off}}, \lambda\right)$, which results in the set of Eqs. (19). The notation $g_{X}$ designates the marginalization of $h\left(\sigma, X_{\text {off }}, \lambda\right)$ over the variable $X$ :

$g_{\lambda}\left(\sigma, X_{\text {off }}\right)=\int h\left(\sigma, X_{\text {off }}, \lambda\right) \mathrm{d} \lambda$,

$g_{X_{\mathrm{off}}}(\sigma, \lambda)=\int h\left(\sigma, X_{\mathrm{off}}, \lambda\right) \mathrm{d} X_{\mathrm{off}}$,

$g_{\sigma}\left(X_{\mathrm{off}}, \lambda\right)=\int h\left(\sigma, X_{\mathrm{off}}, \lambda\right) \mathrm{d} \sigma$.

Integrating again, we obtain

$$
\begin{aligned}
f_{X_{\mathrm{off}}, \lambda}(\sigma) & =\int g_{\lambda}\left(\sigma, X_{\mathrm{off}}\right) \mathrm{d} X_{\mathrm{off}}=\int g_{X_{\mathrm{off}}}(\sigma, \lambda) \mathrm{d} \lambda, \\
f_{\sigma, \lambda}\left(X_{\mathrm{off}}\right) & =\int g_{\lambda}\left(\sigma, X_{\mathrm{off}}\right) \mathrm{d} \sigma=\int g_{\sigma}\left(X_{\mathrm{off}}, \lambda\right) \mathrm{d} \lambda, \\
f_{\sigma, X_{\mathrm{off}}}(\lambda) & =\int g_{X_{\mathrm{off}}}(\sigma, \lambda) \mathrm{d} \sigma=\int g_{\sigma}\left(X_{\mathrm{off}}, \lambda\right) \mathrm{d} X_{\text {off }} .
\end{aligned}
$$

The functions $f, g, h$ are thus linked by derivatives as

$$
\begin{aligned}
g(X, Y) & =\frac{\partial f(X)}{\partial Y}, \\
h(X, Y, Z) & =\frac{\partial^{2} f(X)}{\partial Y \partial Z}=\frac{\partial g(X, Y)}{\partial Z},
\end{aligned}
$$

where $X, Y, Z$ are permutations of the variables $\sigma, X_{\text {off }}, \lambda$. With this method we recover the multiplicity function $f(\sigma)$, which in this notation is $f_{X_{\mathrm{off}}, \lambda}(\sigma)$. This allows us to study the behavior of the dark matter halo mass function according to different variables, making sure that in the end our analysis provides an accurate multiplicity function.

\subsection{Mass-offset-spin function $h\left(\sigma, X_{\text {off }}, \lambda\right)$}

Here we present a model for the generalized mass function $h\left(\sigma, X_{\mathrm{off}}, \lambda\right)$ introduced in the previous subsection. The relaxation state of a dark matter halo is related to the values of $X_{\mathrm{off}}$ and $\lambda$ parameters. We consider HMD, BIGMD, and MDPL 2 to build a 3D histogram of halo counts in bins of $\sigma, X_{\text {off }}, \lambda$, according to Eqs. (17) and (18).

Mass functions are expressed as multiplicity functions $f(\sigma)$. It allows the inclusion of part of the redshift evolution in $\sigma$. We focus on the high-mass end of the mass function, using haloes with $M>2.7 \times 10^{13} M_{\odot} h^{-1}$.

We measure the halo number density in bins of $\log _{10} \sigma^{-1}$ instead of mass. We consider linear spaced bins from -0.09 to 0.6 , with 0.01 width, corresponding to values close to $2.8 \times 10^{13}$ and $1.3 \times 10^{16} M_{\odot} h^{-1}$. We consider 50 bins spanning logarithmically from $10^{-3.8}$ to $10^{-0.2}$ for $X_{\text {off }}$ and 50 bins spanning logarithmically from $10^{-4.5}$ to $10^{-0.1}$ for $\lambda$. This is almost three orders of magnitude higher than the mass resolution in HMD $\left(7.9 \times 10^{10} M_{\odot} h^{-1}\right)$. Therefore, our results will not be impacted by the mass resolution of the simulations. The total sample consists of 8051654 haloes for HMD, 2103896 for BigMD and 142527 for MDPL. First, we estimate directly $f(\sigma)$ for each simulation, according to Eqs. (15) and (16). Then, we estimate $h$ by computing a 3D histogram in the same mass bins and different $X_{\text {off }}$ and $\lambda$ bins.

Uncertainties on histogram values are computed considering a Poisson number count term and a cosmic variance term:

$$
\begin{aligned}
\delta h\left(\sigma, X_{\mathrm{off}}, \lambda\right) & =h\left(\sigma, X_{\mathrm{off}}, \lambda\right) \sqrt{\frac{1}{N_{M, X o f f, \lambda}}+C^{2},} \\
\delta \log _{10} h\left(\sigma, X_{\mathrm{off}}, \lambda\right) & =\frac{1}{\ln 10} \frac{\delta h\left(\sigma, X_{\mathrm{off}}, \lambda\right)}{h\left(\sigma, X_{\mathrm{off}}, \lambda\right)} .
\end{aligned}
$$

Here $C$ is a term accounting for cosmic variance, which is set differently according to the type of simulation. Values for the cosmic variance are given in Table 3 . These values are estimated by Comparat et al. (2017), using a jackknife method for variance at low masses. We fitted bins containing more than 50 haloes, which according to Eq. (22) means an uncertainty of around $15 \%$; in this way our measurement is not dominated by Poisson uncertainty.

\section{Model}

We create a single model for the $h\left(\sigma, X_{\text {off }}, \lambda\right)$ function. We describe in detail its features at $z=0$ and its redshift evolution.

\subsection{Redshift zero}

We consider that both $\lambda$ and $X_{\text {off }}$ probability density functions are described by a modified Schechter function (Eqs. (12) and (14)), and combine these two functions with the multiplicity function along the mass axis. We obtain the following model,

$h\left(\sigma, X_{\mathrm{off}}, \lambda, z, A, a, q, \mu, \alpha, \beta, \gamma, \delta, e\right)=\ldots$

$A \sqrt{\frac{2}{\pi}}\left(\sqrt{a} \frac{\delta_{c}}{\sigma}\right)^{q} \exp \left[-\frac{a}{2} \frac{\delta_{c}^{2}}{\sigma^{2}}\right]\left(\frac{X_{\text {off }}}{\mu^{\prime}}\right)^{\alpha} \ldots$

$\exp \left[-\left(\frac{X_{\mathrm{off}}}{\mu^{\prime}}\right)^{0.05 \alpha}\right]\left(\frac{\lambda}{\mu}\right)^{\gamma} \exp \left[-\left(\frac{X_{\mathrm{off}}}{\mu^{\prime} \sigma^{e}}\right)^{\beta}\left(\frac{\lambda}{\mu}\right)^{\delta}\right]$,

with $\mu^{\prime}=10^{1.83 \log _{10} \mu}$, to disentangle the degeneracy between the two knees of the modified Schechter functions.

This model recalls the Bhattacharya et al. (2011) formulation along the mass axis and considers the combination of a power law and an exponential cutoff (i.e., a modified Schechter function) along the $X_{\text {off }}$ and $\lambda$ axes. The last exponential contains crossed terms between $X_{\text {off }}$ and $\lambda$, which takes into account their correlation. Both $X_{\text {off }}$ and $\lambda$ modified Schechter functions do not have mass dependency (as suggested by Eqs. (12) and (14)). In the Bhattacharya et al. (2011) formulation, there is a double power law; here we consider a single $\sigma$ power law. Additional $\sigma$ dependencies are described by crossed mass-dependent terms in the exponential cutoff, which relates $X_{\text {off }}$ and $\lambda$ modified Schechter functions. The position of the knee of the $X_{\text {off }}$ function (i.e., $\mu^{\prime}$ ) is the same in its two exponential cutoffs, but in the second one we introduce the scaling with mass through the parameter $e$. We correlate directly the knees of the modified Schechter functions for $\lambda$ and $X_{\text {off }}\left(\mu, \mu^{\prime}=10^{1.83 \log _{10} \mu}\right)$, and the slopes of the power law and exponential cutoff of $X_{\mathrm{off}}(\alpha, 0.05 \alpha)$, to write the model in the most compact way possible.

\subsection{Evolution with redshift}

Since structures accrete matter with time and grow, the mass function depends on redshift (Springel et al. 2005). Part of this 
Table 3. Cosmic variance in different MD simulations.

\begin{tabular}{lc}
\hline \hline Simulation & Cosmic variance \\
\hline HMD & 0.02 \\
BigMD & 0.03 \\
MDPL & 0.04 \\
\hline
\end{tabular}

redshift evolution is in the mass- $\sigma$ relation through the matter power spectrum (Eq. (1)). However, this does not make the mass function completely universal at different times. Tinker et al. (2008) showed that a spherical overdensity mass function evolves up to $30 \%$ from $z=0$ to $z=2.5$. Despali et al. (2016) highlighted how only virial overdensity nears the universality for the mass function. Crocce et al. (2010) considered a FOF mass function and found a $10 \%$ evolution up to $z=2$. In this work we use ROCKSTAR to identify haloes, its base is a FOF algorithm as well.

Departures from universality are partially explained by the cosmology dependence of the mass function on the power spectrum and growth rate (Ondaro-Mallea et al. 2021). We find that the distribution functions of $X_{\text {off }}$ and $\lambda$ show a redshift trend as well (see Sect. 4, and Eqs. (12) and (14)). We provide a detailed description of the evolution of $h\left(\sigma, X_{\text {off }}, \lambda\right)$ with redshift. Further investigation, using simulations in different cosmologies, is needed to assess a possible relation between parameters in Eq. (24) and cosmological parameters.

Given our model at $z=0$, we use the latter as the benchmark model to fit the halo mass $-X_{\text {off }}-\lambda$ function at a higher redshift. For this goal we concatenate again samples from HMD, BIGMD, and MDPL2 at redshifts $0.045,0.117,0.221,0.425,0.523,0.702$, $0.779,1.032$, and 1.425 . We note that BIGMD is not tabulated at exactly the same redshift snapshots as the other two simulations. Nonetheless, we use snapshots that are as close as possible, resulting in a $1.3 \%$ difference for the worst-case scenario at $z=1.425$. Further details about the snapshots are available in Appendix A. For all these snapshots we consider the same $X_{\text {off }}$ and $\lambda$ binning as we did for $z=0$. However, we shift the $\sigma$ binning slightly upward compared to the $z=0$ case, which allows us to reach masses of $7 \times 10^{12} M_{\odot} h^{-1}$ at $z=0.702$ and $10^{12} M_{\odot} h^{-1}$ at $z=1.425$.

We include a redshift evolution for all the parameters $A, a, q$, $\mu, \alpha, \beta, \gamma, \delta$, and $e$. We note that we did not consider an evolving critical density contrast with redshift, fixing it at $z=0$. So $\delta_{c}$ in Eq. (23) is fixed at the value of 1.68647. Considering its evolution, even if tiny, introduces the need for additional evolution of the parameters, as pointed out by Bhattacharya et al. (2011). We model the redshift evolution for these parameter using exponents $k_{0}, k_{1}, k_{2}, k_{3}, k_{4}, k_{5}, k_{6}, k_{7}$, and $k_{8}$ as follows in Eq. (24):

$$
\begin{aligned}
\log _{10} A(z) & =\log _{10} A_{0}(1+z)^{k_{0}}, \\
a(z) & =a_{0}(1+z)^{k_{1}}, \\
q(z) & =q_{0}(1+z)^{k_{2}} \\
\log _{10} \mu(z) & =\log _{10} \mu_{0}(1+z)^{k_{3}}, \\
\alpha(z) & =\alpha_{0}(1+z)^{k_{4}}, \\
\beta(z) & =\beta_{0}(1+z)^{k_{5}}, \\
\gamma(z) & =\gamma_{0}(1+z)^{k_{6}}, \\
\delta(z) & =\delta_{0}(1+z)^{k_{7}}, \\
e(z) & =e_{0}(1+z)^{k_{8}} .
\end{aligned}
$$

\section{Results}

We present the result of the fits to the data (Sect. 3) and the parameters of the model (Sect. 6). We fit directly $\log _{10}$ $h\left(\sigma, X_{\mathrm{off}}, \lambda\right)$, which allows better modeling of the high-mass end. We consider a Gaussian likelihood

$\log \mathcal{L}=-0.5 \sum\left(\frac{D-M}{E}\right)^{2}$

where $D$ is $\log _{10} h\left(\sigma, X_{\text {off }}, \lambda\right)$ computed from Eq. (17), $M$ is the base 10 logarithm of the model (Eq. (23)), and $E$ is the uncertainty of $\log _{10} h\left(\sigma, X_{\mathrm{off}}, \lambda\right)$ (see log error in Eq. (22)).

\subsection{Redshift zero}

The best-fit parameters at $z=0$ are obtained maximizing the likelihood in Eq. (25). We derive posterior probability distributions and the Bayesian evidence with the nested sampling Monte Carlo algorithm MLFriends (Buchner 2016, 2019), using the UltraNest ${ }^{1}$ software. The results are shown in Fig. A.1. We used flat priors. The description of the parameters, priors, and posteriors is summarized in Table A.4. We obtain $\log _{10} A=$ $-22.004 \pm 0.006, a=0.885 \pm 0.004, q=2.284 \pm 0.016$, $\log _{10} \mu=-3.326 \pm 0.001, \alpha=5.623 \pm 0.002, \beta=-0.391 \pm 0.001$, $\gamma=3.024 \pm 0.003, \delta=1.209 \pm 0.001, e=-1.105 \pm 0.005$. The parameter $a$ is in agreement with Bhattacharya et al. (2011). Our $q$ parameter shows higher values, but this is expected because an additional mass trend is described by the knee of the exponential cutoff with mixed $X_{\text {off }}, \lambda$ terms. The parameters $\alpha$ and $\gamma$ describe the power-law increment from small $X_{\text {off }}$ and $\lambda$ values, respectively. The second is similar to the values computed for the spin distribution in Sect. 4, while the first is bigger than almost a factor of two. This is expected because an additional offset trend is described by the negative $\beta$ parameter. Moreover, together with $\beta$, the parameter $e$ accounts for the relation between offset and spin, including mass dependency as well. The negative $e$ allows the shifting of the peak along the $X_{\text {off }}$ axis to higher values with mass, according to the findings in Sect. 4.

Since this is a 3D model, we show in Fig. 8 all six combinations of $h\left(\sigma, X_{\text {off }}, \lambda\right)$ integrated in 1D (Eq. (19)). To perform the integrals we exploit the Simpson's rule method for numerical integration ${ }^{2}$. We show each 2D distribution in five different slices of a single quantity. We recover the typical exponential cutoff along the mass axis, as well as the modified Schechter shapes for offset and spin. Our model describes the $\sigma$ and $\lambda$ evolution very well, $g_{X_{\text {off }}}(\sigma, \lambda)$ also agrees with the data in the tails of the distribution. We note the small deviations in the distribution of $X_{\text {off }}$ when $X_{\text {off }}$ values approach the spatial resolution limit. For lowredshift samples, each version of MultiDark has its own resolution limit: $25 \mathrm{kpc} h^{-1}$ for HMD, $10 \mathrm{kpc} h^{-1}$ for BIGMD, $5 \mathrm{kpc} h^{-1}$ for MDPL2. The mass trend of the low $X_{\text {off }}$ slice is slightly underpredicted by the model at low mass. The same holds for the spin trend; there is a $0.1 \mathrm{dex}, 3.1 \sigma$ tension between the peak values of model and data. This is expected within the resolution limit. An improvement toward efficient computation and future generation of $N$-body simulations will be needed to probe the kiloparsec scales of Dark Matter haloes in simulation cubes of the gigaparsec scale. All the panels in Fig. 8 involving $\sigma$ show different uncertainties between succeeding mass bins as a result of the concatenation of bins from different MultiDark versions. MDPL2 bins contain fewer haloes than BIGMD, which contain

\footnotetext{
1 https://johannesbuchner.github.io/UltraNest/

https://docs.scipy.org/doc/
} 
R. Seppi et al.: The mass function dependence on the dynamical state of dark matter haloes


Fig. 8. Single integration of the 3D model. In each panel straight lines indicate the best-fit model, while shaded areas represent the data with $1 \sigma$ uncertainties. Top left panel: $g_{\lambda}\left(\sigma, X_{\text {off }}\right)$ as a function of $X_{\text {off }}$ in different mass slices. Top right panel: $g_{\lambda}\left(\sigma, X_{\text {off }}\right)$ as a function of $\sigma$ in different $X_{\text {off }}$ slices. Middle left panel: $g_{X_{\mathrm{off}}}(\sigma, \lambda)$ as a function of $\lambda$ in different mass slices. Middle right panel: $g_{X_{\mathrm{off}}}(\sigma, \lambda)$ as a function of $\sigma$ in different $\lambda$ slices. Bottom left panel: $g_{\sigma}\left(X_{\mathrm{off}}, \lambda\right)$ as a function of $\lambda$ in different $X_{\text {off }}$ slices. Bottom right panel: $g_{\sigma}\left(X_{\mathrm{off}}, \lambda\right)$ as a function of $X_{\text {off }}$ in different $\lambda$ slices. The integrals are defined in Eq. (19). 

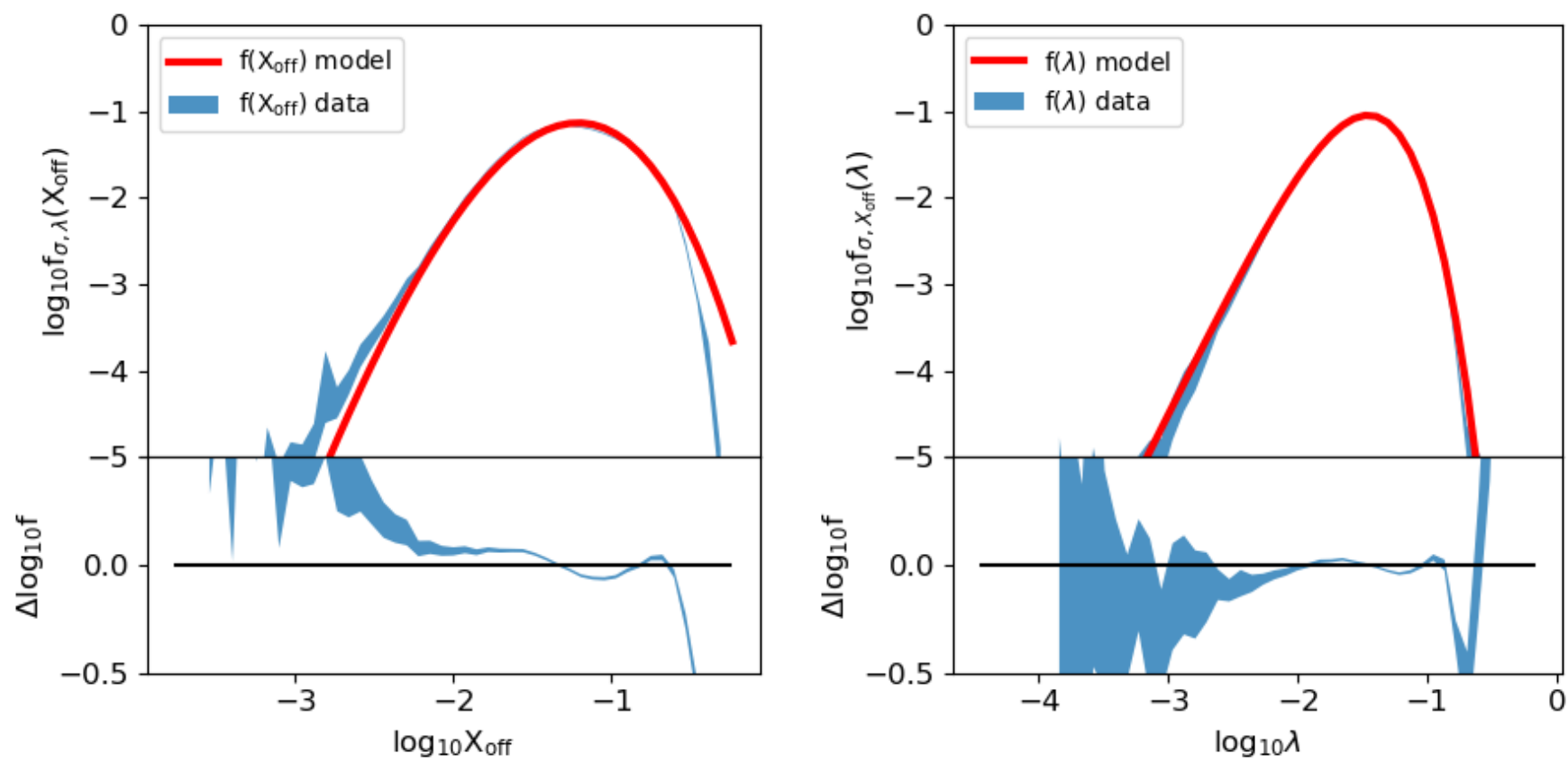

Fig. 9. Comparison between data and model of $f_{\sigma, \lambda}\left(X_{\text {off }}\right)$ and $f_{\sigma, X_{\text {off }}}(\lambda)$. Top panels: the straight red lines indicate the integral on the best-fit model, while the shaded blue areas represent the integral on the $3 \mathrm{~d} h\left(\sigma, X_{\text {off }}, \lambda\right)$ data with $1 \sigma$ uncertainties. Each bottom panel shows the residual trend with $\sigma$ error; the straight black line represents the perfect match between data and model with null residual. Top left panel: $f\left(X_{\text {off }}\right)$ as a function of $X_{\text {off }}$. Bottom left panel: residual between $f_{\sigma, \lambda}\left(X_{\text {off }}\right)$ data and model in logarithmic scale. Top right panel: $f(\lambda)$ as a function of $\lambda$. Bottom right panel: residual between $f_{\sigma, X_{\mathrm{off}}}(\lambda)$ data, and model in logarithmic scale.

fewer haloes than HMD. This translates into smaller uncertainties for bins containing a higher number of haloes. Overall, the model provides an excellent representation of the data.

We further integrate the model in Eq. (23), obtaining the distributions of $X_{\text {off }} f_{\sigma, \lambda}\left(X_{\text {off }}\right)$ and $\lambda f_{\sigma, X_{\text {off }}}(\lambda)$ (Eq. (20)). Figure 9 shows the result. The distributions around the peaks are well described by the model. This is important because these functions are dominated by objects described by the peak of the PDF. The spin distribution is better behaved than the $X_{\text {off }}$ in the tails. This is expected due to spatial resolution limits. Moreover, this is a further confirmation of the fact that $X_{\text {off }}, \lambda$ scatter around their mean values with modified Schechter distributions (see Sect. 4).

We obtain the multiplicity function $f_{X_{\mathrm{off}}, \lambda}(\sigma)$ marginalizing $h\left(\sigma, X_{\mathrm{off}}, \lambda\right)$ on $X_{\mathrm{off}}, \lambda$ (i.e., performing the double integral):

$f_{X_{\text {off }}, \lambda}(\sigma)=\iint h\left(\sigma, X_{\text {off }}, \lambda\right) \mathrm{d} X_{\text {off }} \mathrm{d} \lambda$.

The result is shown in Fig. 10. In the top panel, we show a comparison between our model, the data obtained from simulations, and the Comparat et al. (2017) model, fitted on these same simulations at $z=0$. The multiplicity functions computed directly on each simulation cube, without taking $X_{\text {off }}$ and $\lambda$ into account, are shown by three shaded regions in different colors: red for MDPL2, green for BIGMD, and orange for HMD. Bigger simulation boxes extend to higher mass values. The light blue shaded region represents the $2 \mathrm{D}$ integral computed on the concatenated sample of all three simulations. The solid blue line is the integral of our model and the dashed pink line is the Comparat et al. (2017) model. In the lower panel, we show the percentage difference between the multiplicity function $f(\sigma)$ we recover and the Comparat et al. (2017) model, obtained on the same MultiDark simulations. This difference is always under $3.3 \%$ in the mass range of interest. It is also compatible with uncertainty on the data. Our model is able to recover the halo mass distribution in the simulations, with the advantage of taking into account parameters that describe the dynamical state as well. Once again, we note that our model is adapted to masses higher than $2.7 \times 10^{13} M_{\odot} h^{-1}$ at $z=0\left(10^{12} M_{\odot} h^{-1}\right.$ at $\left.z=1.4\right)$.

\subsection{Evolution with redshift}

To study the redshift evolution we start from the fiducial model at $z=0$. We add the redshift dependence (Eq. (24)) to the best-fit parameters in Eq. (23) at $z=0$. We concatenate samples for ten redshift values, as described in Sect. 6.2.

We obtain the values of the exponents in Eq. (24) fitting the $z$ trend of each parameter for all the concatenated snapshots simultaneously. We obtain $k_{0}=-0.0441 \pm 0.0001, k_{1}=$ $-0.161 \pm 0.001, k_{2}=0.041 \pm 0.002, k_{3}=-0.1286 \pm 0.0002$, $k_{4}=0.1081 \pm 0.0002, k_{5}=-0.311 \pm 0.001, k_{6}=0.0902 \pm 0.0004$, $k_{7}=-0.0768 \pm 0.0004$, and $k_{8}=0.612 \pm 0.002$. The full result is shown by the triangular plot in Fig. A.2. Priors and posteriors for each parameter are given in Table A.5.

Redshift dependence is shown in Fig. 11. The shaded areas include the uncertainty on the best-fit parameter at $z=0$ and on the $z$ evolution, according to Eq. (26)

$\delta P=\left[\left(\frac{\partial P}{\partial P_{0}} \delta P_{0}\right)^{2}+\left(\frac{\partial P}{\partial k} \delta k\right)^{2}\right]^{1 / 2}$,

where $P$ is each parameter in Eq. (23), $P_{0}$ is its value at $z=0$, and $k$ indicates each parameter describing the evolution in Eq. (24).

The parameters $A, q, \mu, \alpha, \gamma$, and $\beta$ show an increasing redshift trend. On the other hand $a, \beta$, and $e$ decrease with redshift. This means that with increasing redshift the modified Schechter functions need to increase more quickly and decrease more slowly. The knee describing the mass trend (parameter $a$ ) decreases with redshift, in agreement with Bhattacharya et al. (2011). They find no redshift dependence for the slope of mass 


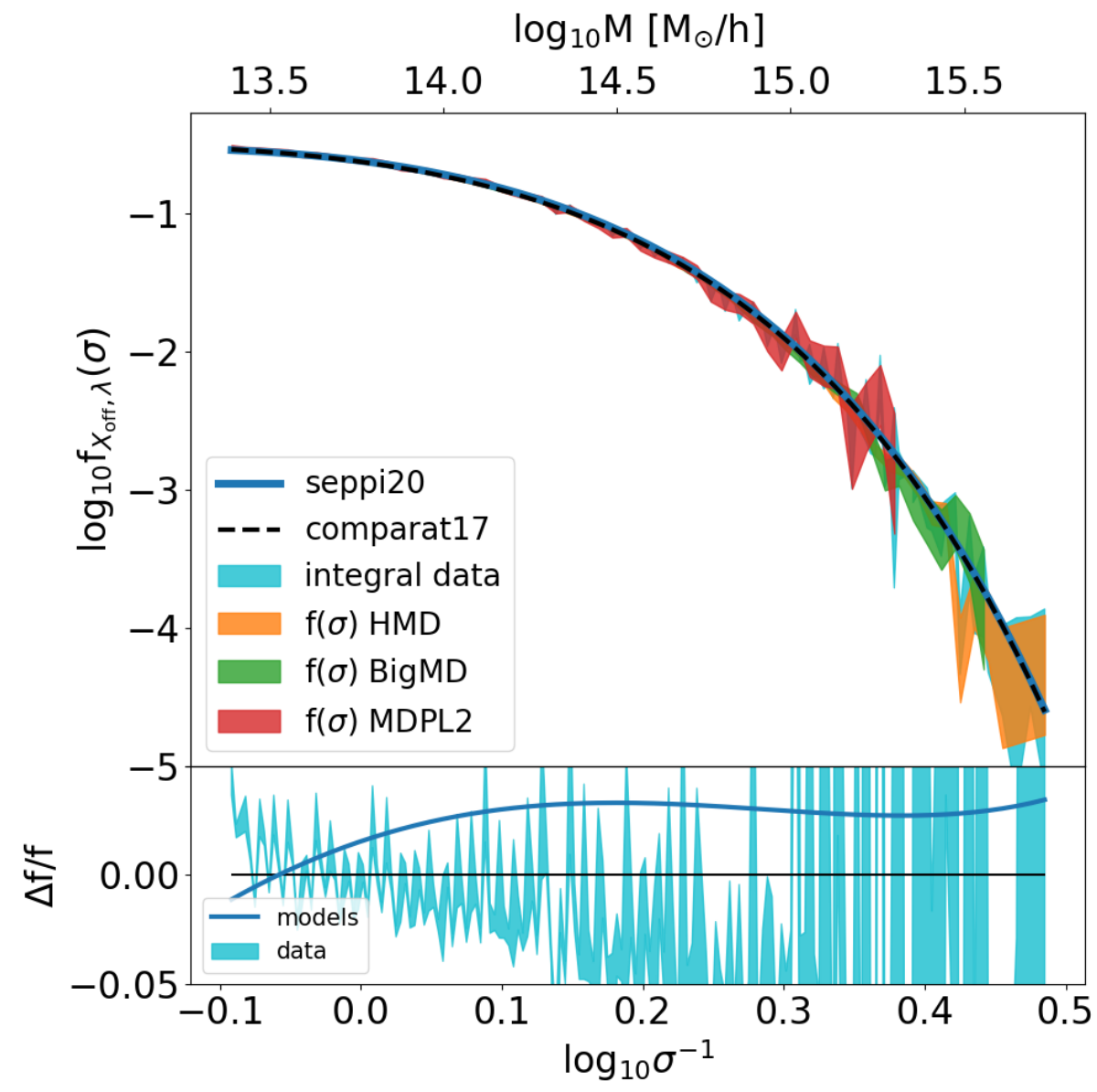

Fig. 10. Comparison of multiplicity functions. Top panel: the three shaded regions show the $1 \sigma$ contours of $f(\sigma)$ data directly computed on different simulations (orange for HMD, green for BIGMD, red for MDPL2). The light blue shaded region is the $1 \sigma$ contour of the $2 \mathrm{D}$ integral computed on the concatenated sample containing all three simulations; the dashed pink line indicates the mass function from Comparat et al. (2017); the blue solid line is the $f(\sigma)$ recovered by integrating our model along $X_{\text {off }}$ and $\lambda$. Bottom panel: the blue thick line is the fractional difference between our $f(\sigma)$ and that of Comparat et al. (2017). The light blue shaded area denotes the $1 \sigma$ contours of the residual between the integrated data and our best-fit model; the black horizontal line indicates the perfect match with null residual. trend (parameter $q$ ). This is not true for this work, where $q$ increases with $z$. However, this is mitigated by the mass trend of the position of the knee in the crossed $X_{\text {off }}, \lambda$ exponential cutoff, which decreases at early times. The fact that the position of the $X_{\text {off }}$ knee (parameter $\mu$ ) moves to higher values at high $z$ confirms the results of Sect. 4, with the higher average value of $X_{\mathrm{off}}$ early in time (Figs. 6 and 7).

\section{Summary and conclusions}

In the context of the hierarchical model of structure formation, the evolution of the number density of galaxy clusters is a powerful cosmological probe. In order to achieve precision cosmology with the next generation of galaxy clusters samples (such as the eROSITA All Sky Survey; Merloni et al. 2012), precise modeling of the theoretical mass function is necessary. We calibrated a model that includes a dynamical description of dark matter haloes. Using the formalism described here and the MultiDark simulations, we quantified the impact on the mass function of the lack of unrelaxed structures (see Fig. 1). We explored relations between quantities that describe different aspects of dark matter haloes, including their dynamical state. We investigated the concentration-mass relation. We confirmed the recent discovery of the concentration upturn at high masses, in agreement with previous results from Prada et al. (2012) and Klypin et al. (2016), based on a similar set of MultiDark simulations. In addition, our model provides a prediction of concentration according to mass and redshift with one single equation (Eq. (9)). The probability density function of concentration is a modified Schechter law, with mass dependency (Eq. (10)). We find that the concentration of low-mass haloes has a faster redshift evolution than high-mass objects, especially in the high-concentration regime. For concentration $c=8$, the PDF for high-mass haloes shifts by $0.32 \mathrm{dex}$ from $z=1.43$ to $z=0$, while the low-mass value changes by 0.49 dex. We find the spin parameter $\lambda$ to be modeled by a linear relation with mass and a probability density function well described by a modified Schechter function (Eq. (12)), in agreement with Rodriguez-Puebla et al. (2016). The offset parameter evolves with mass and redshift according to Eq. (13). The negative slope of the relation suggests that low-mass haloes are typically more relaxed compared to high-mass objects. This is true at every redshift. The offset distribution around the mean value is well described by a modified Schechter function (Eq. (14)). The peak of the distribution shifts by a factor of 1.5 between $z \sim 1.4$ and $z=0$. This is in agreement with haloes relaxing with time and the recent formation of cool cores in galaxy clusters.

We define a general mass function framework, where dark matter haloes are not only described as a function of mass but also by the two additional variables $X_{\text {off }}, \lambda$. This approach considers the mass, offset parameter, and spin of each halo at the same time in a $\sigma-X_{\text {off }}-\lambda$ function (Eq. (18)). We model it in Sect. 6 combining terms of a fiducial mass function (Bhattacharya et al. 2011) with modified Schechter functions for $X_{\text {off }}, \lambda$, as obtained in Sect. 4. This new approach accounts for the dynamical state of dark matter haloes directly in the context of the halo mass function, providing $2 \mathrm{D}$ and $1 \mathrm{D}$ distributions at the same time.

We describe the fitting procedure and results in Sect. 7. Our result at $z=0$ recovers the Comparat et al. (2017) mass function, which is fitted on the same set of simulations, with $3.3 \%$ accuracy. This means that our model is able to account for 

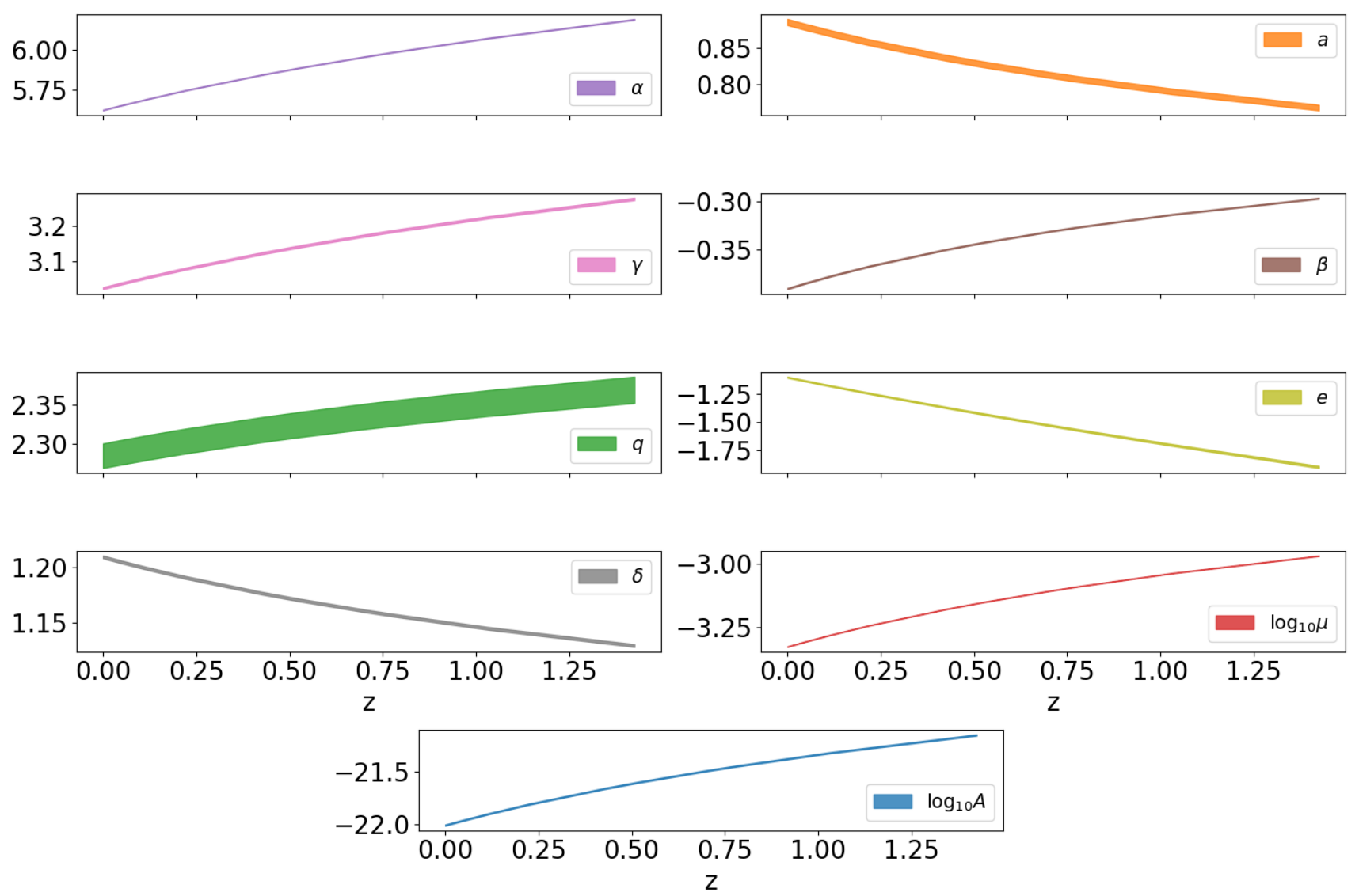

Fig. 11. Redshift evolution of the best-fit parameters of our model. Each panel shows a single parameter. The values at $z=0$ are reported in Table A.4. The redshift evolution is described by Eq. (24); the slopes are given in Table A.5.

the dynamical state of dark matter haloes simultaneously with mass and to describe the multiplicity function with great precision. In addition, our model includes the redshift evolution, according to Eq. (24). The result is shown in Fig. 11.

The link with observations will be explored further in future work.

Acknowledgements. We thank the anonymous referee for the constructive feedback. JC thanks Dominique Eckert for insightful discussions about this project. The CosmoSim database used in this paper is a service by the Leibniz-Institute for Astrophysics Potsdam (AIP). The MultiDark database was developed in cooperation with the Spanish MultiDark Consolider Project CSD2009-00064. The authors gratefully acknowledge the Gauss Centre for Supercomputing e.V. (www.gauss-centre.eu) and the Partnership for Advanced Supercomputing in Europe (PRACE, www .prace-ri.eu) for funding the MultiDark simulation project by providing computing time on the GCS Supercomputer SuperMUC at Leibniz Supercomputing Centre (LRZ, www . lrz . de). We derive posterior probability distributions and the Bayesian evidence with the nested sampling Monte Carlo algorithm MLFriends (Buchner 2016, 2019) using the UltraNest software. To fit relations and distributions in Sect. 4 , we use a $\chi^{2}$ minimization algorithm with CURVE_FIT python package (Virtanen et al. 2020) (https://scipy. org/). In order to perform cosmology calculation, we used the python toolkit CoLossus (Diemer 2018) (https://bdiemer. bitbucket.io/colossus/).

\section{References}

Abazajian, K., Addison, G., Adshead, P., et al. 2019, ArXiv e-prints [arXiv:1907.04473]

Achitouv, I. E., \& Corasaniti, P. S. 2012, JCAP, 2012, 002

Achitouv, I., Wagner, C., Weller, J., \& Rasera, Y. 2014, JCAP, 2014, 077

Allen, S. W., Evrard, A. E., \& Mantz, A. B. 2011, ARA\&A, 49, 409

Angulo, R. E., Baugh, C. M., \& Lacey, C. G. 2008, MNRAS, 387, 921

Angulo, R. E., Springel, V., White, S. D. M., et al. 2012, MNRAS, 426, 2046
Baldi, A. S., De Petris, M., Sembolini, F., et al. 2018, MNRAS, 479, 4028 Baldi, A. S., De Petris, M., Sembolini, F., et al. 2019, J. Phys. Conf. Ser., 1226, 012003

Bartelmann, M. 2010, Class. Quant. Grav., 27, 233001

Baxter, E. J., Sherwin, B. D., \& Raghunathan, S. 2019, JCAP, 2019, 001

Behroozi, P., Wechsler, R., \& Wu, H.-Y. 2013, ApJ, 762, 109

Behroozi, P., Knebe, A., Pearce, F. R., et al. 2015, MNRAS, 454, 3020

Benson, B. A., Ade, P. A. R., Ahmed, Z., et al. 2014, in SPT-3G: A Next-

Generation Cosmic Microwave Background Polarization Experiment on the South Pole Telescope, SPIE Conf. Ser., 9153, 91531P

Bett, P., Eke, V., Frenk, C. S., et al. 2007, MNRAS, 376, 215

Bhattacharya, S., Heitmann, K., White, M., et al. 2011, ApJ, 732, 122

Bianconi, M., Ettori, S., \& Nipoti, C. 2013, MNRAS, 434, 1565

Bilton, L. E., Hunt, M., Pimbblet, K. A., \& Roediger, E. 2019, MNRAS, 490, 5017

Bocquet, S., Saro, A., Dolag, K., \& Mohr, J. J. 2016, MNRAS, 456, 2361

Bocquet, S., Heitmann, K., \& Habib, S. 2020, ApJ, 901, 5

Bond, J. R., Cole, S., Efstathiou, G., \& Kaiser, N. 1991, ApJ, 379, 440

Bryan, G. L., \& Norman, M. L. 1998, ApJ, 495, 80

Buchner, J. 2016, Stat. Comput., 26, 383

Buchner, J. 2019, PASP, 131, 108005

Cibirka, N., Cypriano, E. S., Brimioulle, F., et al. 2017, MNRAS, 468, 1092

Comparat, J., Prada, F., Yepes, G., \& Klypin, A. 2017, MNRAS, 469, 4157

Contreras, S., Zehavi, I., Padilla, N., et al. 2019, MNRAS, 484, 1133

Cooray, A., \& Chen, X. 2002, ApJ, 573, 43

Correa, C. A., Wyithe, J. S. B., Schaye, J., \& Duffy, A. R. 2015, MNRAS, 452, 1217

Crocce, M., Fosalba, P., Castand er, F. J., \& Gaztañaga, E. 2010, MNRAS, 403, 1353

Croton, D. J., Gao, L., \& White, S. D. M. 2007, MNRAS, 374, 1303

Dawson, K. S., Kneib, J.-P., Percival, W. J., et al. 2016, AJ, 151, 44

de Jong, R. 2011, The Messenger, 145, 14

Del Popolo, A., Pace, F., \& Le Delliou, M. 2017, JCAP, 3, 032

DESI Collaboration (Aghamousa, A., et al.) 2016, ArXiv e-prints [arXiv:1611.00036]

Despali, G., Giocoli, C., Angulo, R. E., et al. 2016, MNRAS, 456, 2486 
Diemer, B. 2018, ApJS, 239, 35

Diemer, B., \& Joyce, M. 2019, ApJ, 871, 168

Diemer, B., \& Kravtsov, A. V. 2015, ApJ, 799, 108

Du, W., Fan, Z., Shan, H., et al. 2015, ApJ, 814, 120

Duffy, A. R., Schaye, J., Kay, S. T., \& Dalla Vecchia, C. 2008, MNRAS, 390, L64

Dutton, A. A., \& Macciò, A. V. 2014, MNRAS, 441, 3359

Eckert, D., Molendi, S., \& Paltani, S. 2011, A\&A, 526, A79

Eckert, D., Ghirardini, V., Ettori, S., et al. 2019, A\&A, 621, A40

Ettori, S., \& Brighenti, F. 2008, MNRAS, 387, 631

Finoguenov, A., Merloni, A., Comparat, J., et al. 2019, The Messenger, 175, 39

Finoguenov, A., Rykoff, E., Clerc, N., et al. 2020, A\&A, 638, A114

Foëx, G., Motta, V., Jullo, E., Limousin, M., \& Verdugo, T. 2014, A\&A, 572, A19

Gao, L., Springel, V., \& White, S. D. M. 2005, MNRAS, 363, L66

Giocoli, C., Tormen, G., \& Sheth, R. K. 2012, MNRAS, 422, 185

Heitmann, K., Frontiere, N., Sewell, C., et al. 2015, ApJS, 219, 34

Henson, M. A., Barnes, D. J., Kay, S. T., McCarthy, I. G., \& Schaye, J. 2017, MNRAS, 465, 3361

Hitomi Collaboration (Aharonian, F., et al.) 2018, PASJ, 70, 9

Hollowood, D. L., Jeltema, T., Chen, X., et al. 2019, ApJS, 244, 22

Hwang, H. S., \& Lee, M. G. 2007, ApJ, 662, 236

Ider Chitham, J., Comparat, J., Finoguenov, A., et al. 2020, MNRAS, 499, 4768

Ishiyama, T., Enoki, M., Kobayashi, M. A. R., et al. 2015, PASJ, 67, 61

Jenkins, A., Frenk, C. S., White, S. D. M., et al. 2001, MNRAS, 321, 372

Käfer, F., Finoguenov, A., Eckert, D., et al. 2019, A\&A, 628, A43

Käfer, F., Finoguenov, A., Eckert, D., et al. 2020, A\&A, 634, A8

Kilbinger, M. 2015, Rep. Prog. Phys., 78, 086901

Klypin, A., Yepes, G., Gottlöber, S., Prada, F., \& Heß, S. 2016, MNRAS, 457, 4340

Knebe, A., Knollmann, S. R., Muldrew, S. I., et al. 2011, MNRAS, 415, 2293

Knebe, A., Pearce, F. R., Lux, H., et al. 2013, MNRAS, 435, 1618

Kravtsov, A. V., Klypin, A. A., \& Khokhlov, A. M. 1997, ApJS, 111, 73

Lang, M., Holley-Bockelmann, K., \& Sinha, M. 2015, ApJ, 811, 152

Laureijs, R., Amiaux, J., Arduini, S., et al. 2011, ArXiv e-prints [arXiv:1110.3193]

LSST Science Collaboration (Abell, P. A., et al.) 2009, ArXiv e-prints [arXiv:0912.0201]

Ludlow, A. D., Navarro, J. F., Li, M., et al. 2012, MNRAS, 427, 1322

Ludlow, A. D., Navarro, J. F., Boylan-Kolchin, M., et al. 2013, MNRAS, 432, 1103

Ludlow, A. D., Navarro, J. F., Angulo, R. E., et al. 2014, MNRAS, 441, 378

Ludlow, A. D., Bose, S., Angulo, R. E., et al. 2016, MNRAS, 460, 1214

Macciò, A. V., Dutton, A. A., \& van den Bosch, F. C. 2008, MNRAS, 391, 1940

Manolopoulou, M., \& Plionis, M. 2017, MNRAS, 465, 2616

McClintock, T., Rozo, E., Becker, M. R., et al. 2019, ApJ, 872, 53
Meneghetti, M., \& Rasia, E. 2013, ArXiv e-prints [arXiv:1303.6158]

Merloni, A., Predehl, P., Becker, W., et al. 2012, ArXiv e-prints [arXiv:1209.3114]

Murray, S. G., Power, C., \& Robotham, A. S. G. 2013, Astron. Comput., 3, 23

Navarro, J. F., Frenk, C. S., \& White, S. D. M. 1996, ApJ, 462, 563

Neto, A. F., Gao, L., Bett, P., et al. 2007, MNRAS, 381, 1450

Nishimichi, T., Takada, M., Takahashi, R., et al. 2019, ApJ, 884, 29

Ondaro-Mallea, L., Angulo, R. E., Zennaro, M., Contreras, S., \& Aricò, G. 2021, ArXiv e-prints [arXiv:2102.08958]

Peebles, P. J. E. 1969, ApJ, 155, 393

Phriksee, A., Jullo, E., Limousin, M., et al. 2020, MNRAS, 491, 1643

Planck Collaboration XI. 2014, A\&A, 571, A11

Poveda-Ruiz, C. N., Forero-Romero, J. E., \& Muñoz-Cuartas, J. C. 2016, ApJ, 832, 169

Prada, F., Klypin, A. A., Cuesta, A. J., Betancort-Rijo, J. E., \& Primack, J. 2012, MNRAS, 423, 3018

Predehl, P., Andritschke, R., Arefiev, V., et al. 2021, A\&A, 647, A1

Press, W. H., \& Schechter, P. 1974, ApJ, 187, 425

Ragagnin, A., Dolag, K., Moscardini, L., Biviano, A., \& D’Onofrio, M. 2019, MNRAS, 486, 4001

Rephaeli, Y. 1995, ARA\&A, 33, 541

Riebe, K., Partl, A. M., Enke, H., et al. 2013, Astron. Nachr., 334, 691

Rodriguez-Puebla, A., Behroozi, P., Primack, J., et al. 2016, MNRAS, 462, 893

Salvati, L., Douspis, M., \& Aghanim, N. 2020, A\&A, 643, A20

Sereno, M., Giocoli, C., Ettori, S., \& Moscardini, L. 2015, MNRAS, 449, 2024

Shan, H., Kneib, J.-P., Li, R., et al. 2017, ApJ, 840, 104

Sheth, R. K., \& Tormen, G. 1999, MNRAS, 308, 119

Sheth, R. K., \& Tormen, G. 2002, MNRAS, 329, 61

Skillman, S. W., Warren, M. S., Turk, M. J., et al. 2014, ArXiv e-prints [arXiv:1407.2600]

Song, H., Hwang, H. S., Park, C., Smith, R., \& Einasto, M. 2018, ApJ, 869, 124 Spergel, D., Gehrels, N., Baltay, C., et al. 2015, ArXiv e-prints [arXiv:1503.03757]

Springel, V. 2005, MNRAS, 364, 1105

Springel, V., White, S. D. M., Jenkins, A., et al. 2005, Nature, 435, 629

Sunyaev, R. A., Norman, M. L., \& Bryan, G. L. 2003, Astron. Lett., 29, 783

Thomas, P. A., Muanwong, O., Pearce, F. R., et al. 2001, MNRAS, 324, 450

Tinker, J., Kravtsov, A. V., Klypin, A., et al. 2008, ApJ, 688, 709

Tovmassian, H. M. 2015, Astrophysics, 58, 328

Umetsu, K. 2020, A\&ARv, 28, 1

van Uitert, E., Gilbank, D. G., Hoekstra, H., et al. 2016, A\&A, 586, A43

Virtanen, P., Gommers, R., Oliphant, T. E., et al. 2020, Nat. Methods, 17, 261

Wang, J., Bose, S., Frenk, C. S., et al. 2020, Nature, 585, 39

Weinberg, D. H., Mortonson, M. J., Eisenstein, D. J., et al. 2013, Phys. Rep, 530, 87

Zhao, D. H., Mo, H. J., Jing, Y. P., \& Börner, G. 2003, MNRAS, 339, 12

Zhao, D. H., Jing, Y. P., Mo, H. J., \& Börner, G. 2009, ApJ, 707, 354

Zubeldia, Í., \& Challinor, A. 2019, MNRAS, 489, 401 


\section{Appendix A: Figures and tables}

In this section we collect the figures and tables relative to this work. They describe the mean relations between concentration, offset parameter, spin, and mass, as well as the full probability density functions of these quantities. Moreover, we show additional plots describing the halo $\sigma-X_{\mathrm{off}}-\lambda$ function.

Table A.1. Best-fit parameters for concentration $-\sigma$ relation and its PDF P(c).

\begin{tabular}{|c|c|c|c|c|c|c|c|}
\hline & & & $a_{0}$ & & $b_{0}$ & & \\
\hline & & & $0.754091 \pm 0.000004$ & & $0.574413 \pm 0.000002$ & & \\
\hline & $A$ & $\alpha$ & $\beta$ & $x_{0}$ & $e_{0}$ & $e_{1}$ & $e_{2}$ \\
\hline$z=0$ & $0.041 \pm 0$ & $1.397 \pm 0.001$ & $2.604 \pm 0.001$ & $7.225 \pm 0.002$ & $0.089 \pm 0.001$ & $0.776 \pm 0.001$ & $-0.959 \pm 0.001$ \\
\hline$z=0.52$ & $0.044 \pm 0$ & $2.501 \pm 0.001$ & $1.283 \pm 0.001$ & $2.394 \pm 0.001$ & $0.579 \pm 0.001$ & $-0.325 \pm 0.001$ & $0.0334 \pm 0.001$ \\
\hline$z=1.03$ & $3.37 \mathrm{e}-3 \pm 0$ & $4.14 \pm 0.12$ & $0.927 \pm 0.002$ & $0.688 \pm 0.025$ & $0.188 \pm 0.002$ & $0.081 \pm 0.001$ & $0.0813 \pm 0.001$ \\
\hline$z=1.43$ & $2.54 \mathrm{e}-3 \pm 0$ & $4.45 \pm 0.13$ & $0.924 \pm 0.002$ & $0.623 \pm 0.031$ & $0.198 \pm 0.003$ & $0.095 \pm 0.001$ & $0.103 \pm 0.001$ \\
\hline
\end{tabular}

Notes. The models are described by Eqs. (9) and (10). Uncertainties are of the percentage accuracy. In order to have compact information, when uncertainties are smaller than 4 order of magnitudes with respect to the parameter, a value of 0 is written.

Table A.2. Best-fit parameters for $\lambda-\sigma$ relation and its PDF $\mathrm{P}(\lambda)$ at different redshifts.

\begin{tabular}{lcccc}
\hline \hline \multicolumn{3}{c}{$a_{0}$} & \multicolumn{3}{c}{$b_{0}$} \\
\hline & $4.5357 \mathrm{e}-2 \pm 2 \mathrm{e}-6$ & & $-5.4328 \mathrm{e}-3 \pm 1 \mathrm{e}-7$ & \\
\hline \multicolumn{1}{c}{$\mathrm{A}$} & $\alpha$ & $\beta$ & $x_{0}$ \\
\hline$z=0$ & $0.274 \pm 0.009$ & $3.002 \pm 0.013$ & $0.773 \pm 0.001$ & $4.33 \mathrm{e}-3 \pm 0$ \\
$z=0.52$ & $1.01 \pm 0.02$ & $2.623 \pm 0.004$ & $0.911 \pm 0.001$ & $7.46 \mathrm{e}-3 \pm 0$ \\
$z=1.03$ & $1.769 \pm 0.009$ & $2.409 \pm 0.002$ & $1.006 \pm 0.001$ & $9.32 \mathrm{e}-3 \pm 0$ \\
$z=1.43$ & $2.089 \pm 0.007$ & $2.351 \pm 0.001$ & $1.031 \pm 0.001$ & $9.34 \mathrm{e}-3 \pm 0$ \\
\hline
\end{tabular}

Notes. The models are described by Eqs. (11) and (12). Uncertainties are the percentage accuracy. In order to have compact information, when uncertainties are smaller than 4 order of magnitudes with respect to the parameter, a value of 0 is written.

Table A.3. Best-fit parameters for $X_{\text {off }}-\sigma$ relation and its PDF $\mathrm{P}\left(X_{\text {off }}\right)$.

\begin{tabular}{lcccc}
\hline \hline & $a_{0}$ & \multicolumn{3}{c}{$b_{0}$} \\
\hline & $-1.30418 \pm 0.00001$ & & $0.15084 \pm 0.00001$ & \\
\hline $\log _{10} A$ & $\alpha$ & $\beta$ & $\log _{10} x_{0}$ \\
\hline$z=0$ & $-3.09 \pm 0.26$ & $3.71 \pm 0.12$ & $0.64 \pm 0.03$ & $-2.31 \pm 0.09$ \\
$z=0.52$ & $-2.72 \pm 0.17$ & $3.69 \pm 0.03$ & $0.69 \pm 0.01$ & $-2.11 \pm 0.01$ \\
$z=1.03$ & $-2.18 \pm 0.05$ & $3.44 \pm 0.02$ & $0.77 \pm 0.01$ & $-1.88 \pm 0.01$ \\
$z=1.43$ & $-1.79 \pm 0.02$ & $3.19 \pm 0.01$ & $0.84 \pm 0.01$ & $-1.70 \pm 0.01$ \\
\hline
\end{tabular}

Notes. The models are described by Eqs. (13) and (14). Uncertainties on the mean relation are under the percentage level accuracy. 
R. Seppi et al.: The mass function dependence on the dynamical state of dark matter haloes

Table A.4. Model parameters with priors and posterior constraints at redshift zero.

\begin{tabular}{lcc}
\hline \hline Parameter & Prior & Posterior \\
\hline $\log _{10} A$ & $(-23,-20)$ & $-22.004_{-0.006}^{+0.006}$ \\
$a$ & $(0.5,1.0)$ & $0.885_{-0.004}^{+0.004}$ \\
$q$ & $(1.5,2.5)$ & $2.284_{-0.016}^{+0.016}$ \\
$\log _{10} \mu$ & $(-3.5,-3.0)$ & $-3.326_{-0.001}^{+0.001}$ \\
$\alpha$ & $(5.4,5.8)$ & $5.623_{-0.002}^{+0.002}$ \\
$\beta$ & $(-0.5,-0.3)$ & $-0.391_{-0.001}^{+0.001}$ \\
$\gamma$ & $(2.8,3.2)$ & $3.024_{-0.003}^{+0.003}$ \\
$\delta$ & $(1.0,1.4)$ & $1.209_{-0.001}^{+0.001}$ \\
$e$ & $(-1.2,-0.8)$ & $-1.105_{-0.005}^{+0.005}$ \\
\hline
\end{tabular}

Notes. The full distribution of the posteriors is shown in Fig. A.1.

Table A.5. Model parameters with prior and posterior constraints for the redshift evolution of the halo $\sigma-X_{\mathrm{off}}-\lambda$ function.

\begin{tabular}{lcc}
\hline \hline Parameter & Prior & Posterior \\
\hline$k_{0}$ & $(-0.08,0.07)$ & $-0.0441 \pm 0.0001$ \\
$k_{1}$ & $(-0.25,0.05)$ & $-0.161 \pm 0.001$ \\
$k_{2}$ & $(-0.05,0.15)$ & $0.041 \pm 0.002$ \\
$k_{3}$ & $(-0.18,0.02)$ & $-0.1286 \pm 0.0002$ \\
$k_{4}$ & $(-0.02,0.18)$ & $0.108 \pm 0.0002$ \\
$k_{5}$ & $(-0.7,0.1)$ & $-0.311 \pm 0.001$ \\
$k_{6}$ & $(-0.1,0.2)$ & $0.0902 \pm 0.0004$ \\
$k_{7}$ & $(-0.2,0.1)$ & $-0.0768 \pm 0.0004$ \\
$k_{8}$ & $(-0.05,0.85)$ & $0.612 \pm 0.002$ \\
\hline
\end{tabular}

Notes. In the posteriors, when the $1 \sigma$ uncertainty on the onedimensional distribution is smaller than 3 order of magnitudes with respect to the parameter, we write null error. The redshift evolution of each parameter is shown in Fig. 11.
Table A.6. Full list of snapshots used, available in HMD, BIGMD, MDPL2.

\begin{tabular}{lccccc}
\hline \hline$a$ & $z$ & $T$ (Gyr) & HMDPL & BIGMD & MDPL2 \\
\hline 1.0 & 0 & 13.82 & $\mathrm{x}$ & $\mathrm{x}$ & $\mathrm{x}$ \\
0.9567 & 0.04526 & 13.19 & $\mathrm{x}$ & & $\mathrm{x}$ \\
0.956 & 0.04603 & 13.18 & & $\mathrm{x}$ & \\
0.8953 & 0.1169 & 12.27 & & $\mathrm{x}$ & \\
0.8951 & 0.1172 & 12.26 & $\mathrm{x}$ & & $\mathrm{x}$ \\
0.8192 & 0.2207 & 11.09 & $\mathrm{x}$ & & $\mathrm{x}$ \\
0.8173 & 0.2235 & 11.06 & & $\mathrm{x}$ & \\
0.7016 & 0.4253 & 9.198 & $\mathrm{x}$ & & $\mathrm{x}$ \\
0.7003 & 0.428 & 9.177 & & $\mathrm{x}$ & \\
0.6583 & 0.5191 & 8.487 & & $\mathrm{x}$ & \\
0.6565 & 0.5232 & 8.458 & $\mathrm{x}$ & & $\mathrm{x}$ \\
0.5876 & 0.7018 & 7.319 & $\mathrm{x}$ & & $\mathrm{x}$ \\
0.5864 & 0.7053 & 7.299 & & $\mathrm{x}$ & \\
0.5623 & 0.7785 & 6.90 & & $\mathrm{x}$ & \\
0.5622 & 0.7787 & 6.89 & $\mathrm{x}$ & & $\mathrm{x}$ \\
0.5 & 1 & 5.88 & & $\mathrm{x}$ & \\
0.4922 & 1.032 & 5.753 & $\mathrm{x}$ & & $\mathrm{x}$ \\
0.4123 & 1.425 & 4.482 & $\mathrm{x}$ & & $\mathrm{x}$ \\
0.409 & 1.445 & 4.431 & & $\mathrm{x}$ & \\
\hline
\end{tabular}



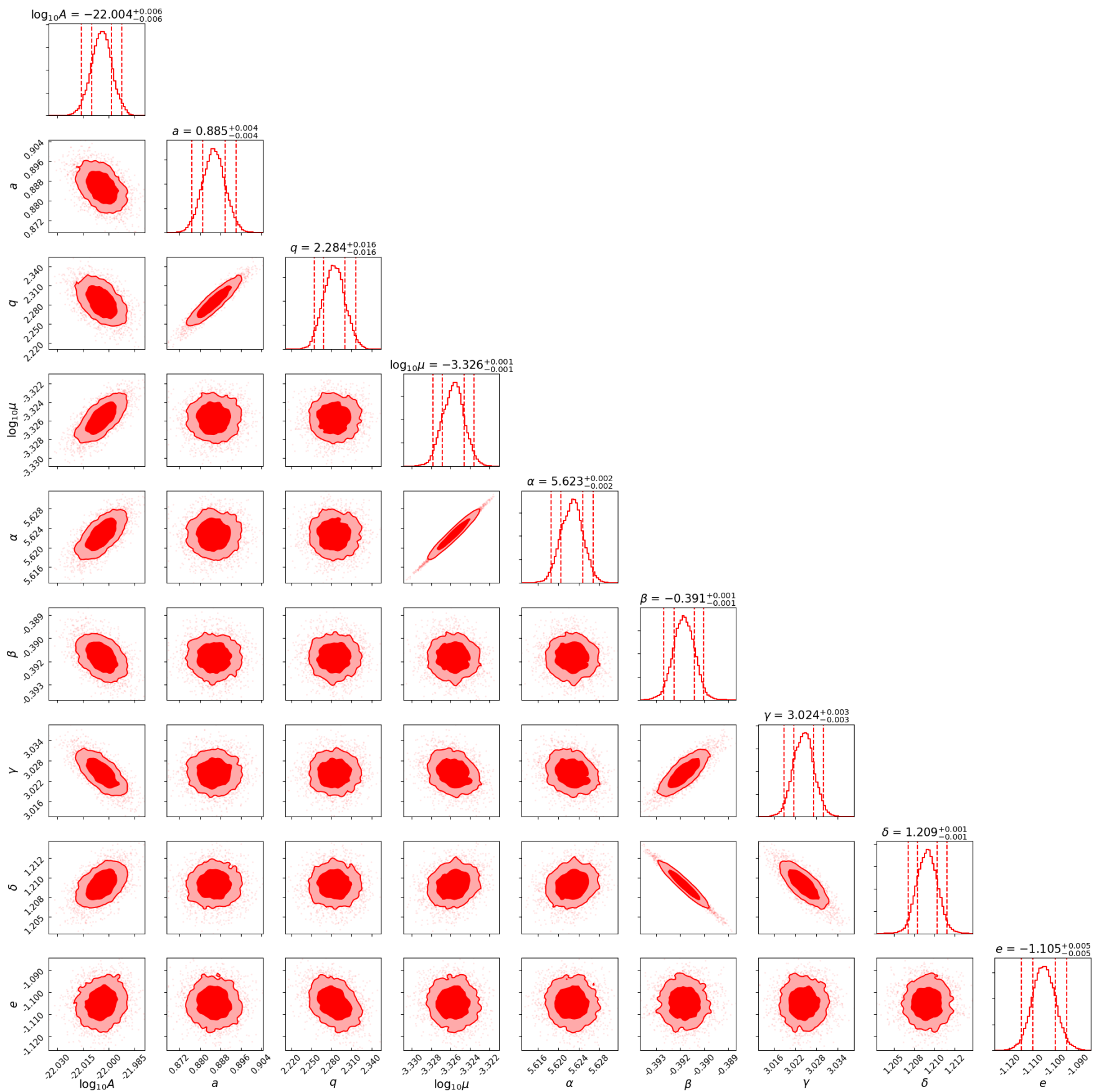

Fig. A.1. Marginalized posterior distributions of the best-fit parameters of the halo $\sigma-X_{\text {off }}-\lambda$ function. The 0.68 and 0.95 confidence levels of the posteriors are shown as filled $2 \mathrm{D}$ contours. The $2.5 \mathrm{th}, 16 \mathrm{th}, 84 \mathrm{th}$, and 97.5 th percentiles of the one-dimensional posterior distributions are indicated by the vertical lines on the diagonal plots. The model is given by Eq. (23). The parameters are also given in Table A.4. 
R. Seppi et al.: The mass function dependence on the dynamical state of dark matter haloes

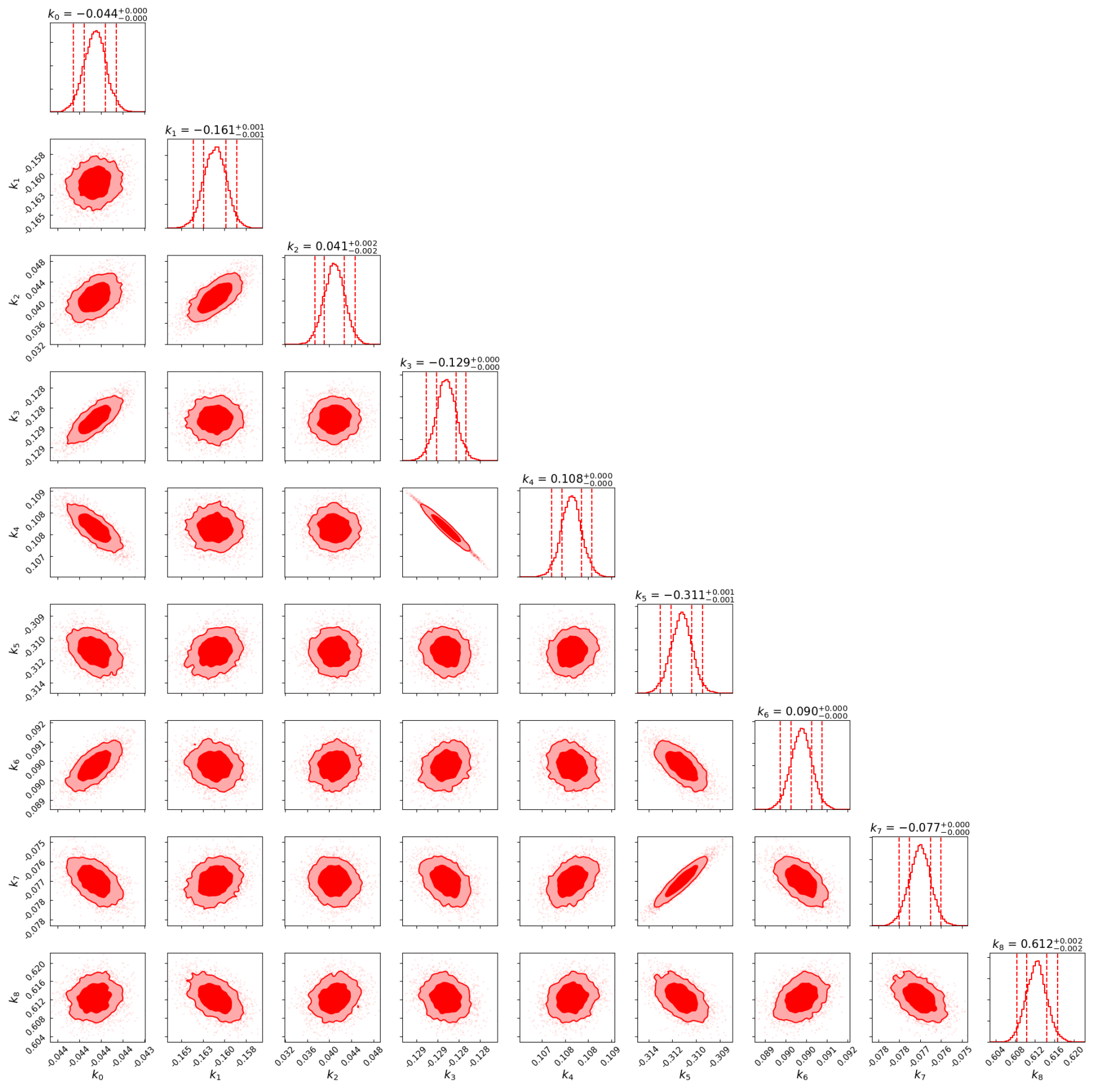

Fig. A.2. Marginalized posterior distributions of the best-fit parameters describing the redshift evolution of $h\left(\sigma, X_{\mathrm{off}}, \lambda\right)$. The 0.68 and 0.95 confidence levels of the posteriors are shown as filled 2D contours. The model is given by Eq. (24). The parameters are reported in Table A.5. 


\section{Appendix B: Offset in physical units}

Here we present the results of the same analysis elaborated in Sects. 4 and 7 to the offset parameter in physical units $X_{\mathrm{off}, P}$, measured in $\mathrm{kpc} h^{-1}$. This approach allows the comparison between the physics of dark matter simulations and observations.

\section{B.1. Offset-mass-redshift relation}

The relation between $X_{\mathrm{off}, P}$, mass and redshift is modeled by

$\log _{10} X_{\mathrm{off}, P}(\sigma, z)=\frac{b_{0}}{E(z)^{0.06}}\left[1+2.39\left(\frac{\sigma}{a_{0} E(z)^{0.8}}\right)^{c_{0} \sigma}\right]$.

The distribution of $X_{\mathrm{off}, P}$ around its mean value is described by a modified Schechter function, but $X_{\text {off, } P}$ is not normalized to the virial radius. Therefore, a mass dependence has to be included in the relation.

$P\left(X_{\mathrm{off}, P}\right)=A\left(\frac{X_{\mathrm{off}, P}}{x_{0} \sigma^{e_{0}}}\right)^{\alpha} \exp \left[-\left(\frac{X_{\mathrm{off}, P}}{x_{0} \sigma^{e_{0}}}\right)^{\beta}\right]$.
The best-fit parameters are given in Table B.1, and the results are shown in Figs. B.1 and B.2.

\section{B.2. Mass $-X_{\mathrm{off}, P}-\lambda$ function}

In this section we collect figures and tables that describe $h\left(\sigma, X_{\mathrm{off}, P}, \lambda\right)$. The analysis is similar to $h\left(\sigma, X_{\mathrm{off}}, \lambda\right)$ explained in Sects. 5 and 6 ; the only difference is that the modified Schechter function that describes $X_{\mathrm{off}, P}$ needs a mass-dependent term. Therefore, we introduce an additional parameter and model the distribution according to

$h\left(\sigma, X_{\mathrm{off}, P}, \lambda, z, A, a, q, \mu, \alpha, \beta, e_{0}, \gamma, \delta, e_{1}\right)=\ldots$

$A \sqrt{\frac{2}{\pi}}\left(\sqrt{a} \frac{\delta_{c}}{\sigma}\right)^{q} \exp \left[-\frac{a}{2} \frac{\delta_{c}^{2}}{\sigma^{2}}\right]\left(\frac{X_{\mathrm{off}, P}}{\mu \sigma^{e_{0}}}\right)^{\alpha} \ldots$

$\exp \left[-\left(\frac{X_{\mathrm{off}, P}}{\mu \sigma^{e_{0}}}\right)^{0.05 \alpha}\right]\left(\frac{\lambda}{0.7 \mu}\right)^{\gamma} \exp \left[-\left(\frac{X_{\mathrm{off}, P}}{\mu \sigma^{e_{1}}}\right)^{\beta}\left(\frac{\lambda}{0.7 \mu}\right)^{\delta}\right]$.

We recover the fiducial mass function at the $\sim 3.9 \%$ level.

Table B.1. Best-fit parameters for $X_{\mathrm{off}, P}-\sigma$ relation and $P\left(X_{\mathrm{off}, P}\right)$.

\begin{tabular}{|c|c|c|c|c|c|}
\hline & & $a_{0}$ & $b_{0}$ & $c_{0}$ & \\
\hline & & $0.16523 \pm 0.00004$ & $0.74872 \pm 0.00001$ & $-0.39607 \pm 0.00003$ & \\
\hline & $A$ & $\alpha$ & $\beta$ & $x_{0}$ & $e_{0}$ \\
\hline$z=0$ & $-5.45 \pm 0.01$ & $10.56 \pm 0.87$ & $1.23 \pm 0.09$ & $2.34 \pm 0.19$ & $-0.57 \pm 0.05$ \\
\hline$z=0.52$ & $-5.45 \pm 0.01$ & $10.58 \pm 0.87$ & $1.23 \pm 0.11$ & $2.35 \pm 0.19$ & $-0.43 \pm 0.04$ \\
\hline$z=1.03$ & $-5.35 \pm 0.01$ & $10.54 \pm 0.86$ & $1.24 \pm 0.11$ & $2.36 \pm 0.19$ & $-0.33 \pm 0.03$ \\
\hline$z=1.43$ & $-5.32 \pm 0.01$ & $10.56 \pm 0.86$ & $1.32 \pm 0.11$ & $2.36 \pm 0.19$ & $-0.46 \pm 0.04$ \\
\hline
\end{tabular}

Notes. The models are described by Eqs. (B.1) and (B.2). Uncertainties on the mean relation are under the percentage level accuracy.

Table B.2. $h\left(\sigma, X_{\mathrm{off}, P}, \lambda\right)$ model parameters with priors and posterior costraints.

\begin{tabular}{lcc}
\hline \hline Parameter & Prior & Posterior \\
\hline $\log _{10} A$ & $(-23,-20)$ & $-22.004_{-0.009}^{+0.009}$ \\
$a$ & $(0.5,1.0)$ & $0.878_{-0.004}^{+0.004}$ \\
$q$ & $(1.5,2.5)$ & $2.257_{-0.013}^{+0.013}$ \\
$\log _{10} \mu$ & $(-3.5,-3.0)$ & $-3.149_{-0.002}^{+0.002}$ \\
$\alpha$ & $(5.4,5.8)$ & $5.624_{-0.002}^{+0.002}$ \\
$\beta$ & $(-0.4,-0.3)$ & $-0.365_{-0.001}^{+0.001}$ \\
$e_{0}$ & $(-2.0,-1.4)$ & $-1.606_{-0.002}^{+0.002}$ \\
$\gamma$ & $(2.8,3.2)$ & $3.095_{-0.003}^{+0.003}$ \\
$\delta$ & $(1.0,1.4)$ & $1.168_{-0.001}^{+0.002}$ \\
$e_{1}$ & $(-3.0,-2.5)$ & $-2.270_{-0.005}^{+0.005}$ \\
\hline
\end{tabular}

Notes. The full distribution of the posteriors is shown in the triangular plot in Fig. B.7. 
R. Seppi et al.: The mass function dependence on the dynamical state of dark matter haloes

Table B.3. Model parameters with prior and posterior constraints for the redshift evolution of $h\left(\sigma, X_{\mathrm{off}, P}, \lambda\right)$.

\begin{tabular}{lcc}
\hline \hline Parameter & Prior & Posterior \\
\hline$k_{0}$ & $(-0.08,0.07)$ & $-0.0131 \pm 0.0001$ \\
$k_{1}$ & $(-0.25,0.05)$ & $-0.146 \pm 0.001$ \\
$k_{2}$ & $(-0.1,0.1)$ & $0.04 \pm 0.002$ \\
$k_{3}$ & $(-0.15,0.05)$ & $-0.0716 \pm 0.0003$ \\
$k_{4}$ & $(-0.05,0.15)$ & $0.0789 \pm 0.0001$ \\
$k_{5}$ & $(-0.7,0.1)$ & $-0.4199 \pm 0.0005$ \\
$k_{6}$ & $(-0.15,0.05)$ & $-0.0554 \pm 0.001$ \\
$k_{7}$ & $(-0.05,0.25)$ & $0.1526 \pm 0.0002$ \\
$k_{8}$ & $(-0.25,0.05)$ & $-0.1834 \pm 0.0004$ \\
$k_{9}$ & $(-0.05,0.35)$ & $0.235 \pm 0.001$ \\
\hline
\end{tabular}

Notes. In the posteriors, when the $1 \sigma$ uncertainty on the onedimensional distribution is smaller than 3 order of magnitudes with respect to the parameter, we write null error. The redshift evolution of each parameter is shown in Fig. B.6.

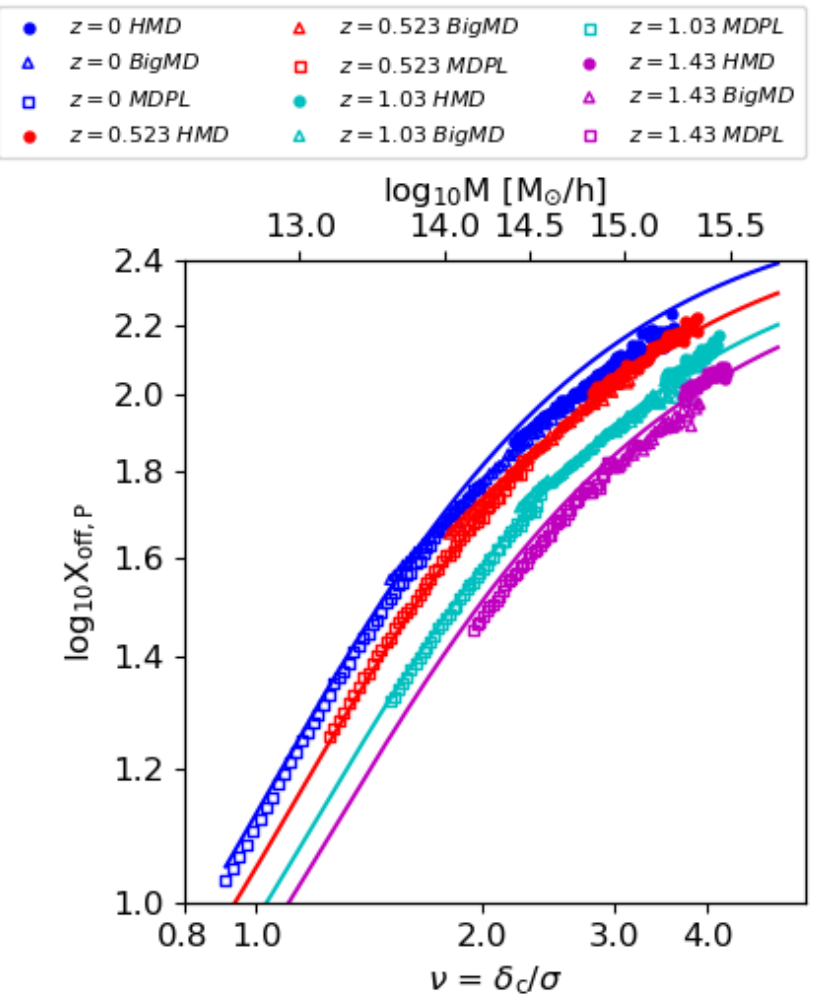

Fig. B.1. $X_{\mathrm{off}, P}-\sigma$ relation (Eq. (B.1)). Circular dots, triangles, and squares represent HMD, BIGMD, and MDPL2, respectively. They are color-coded by redshift. Straight lines show the best-fit model. Parameters are given in Table B.1. 

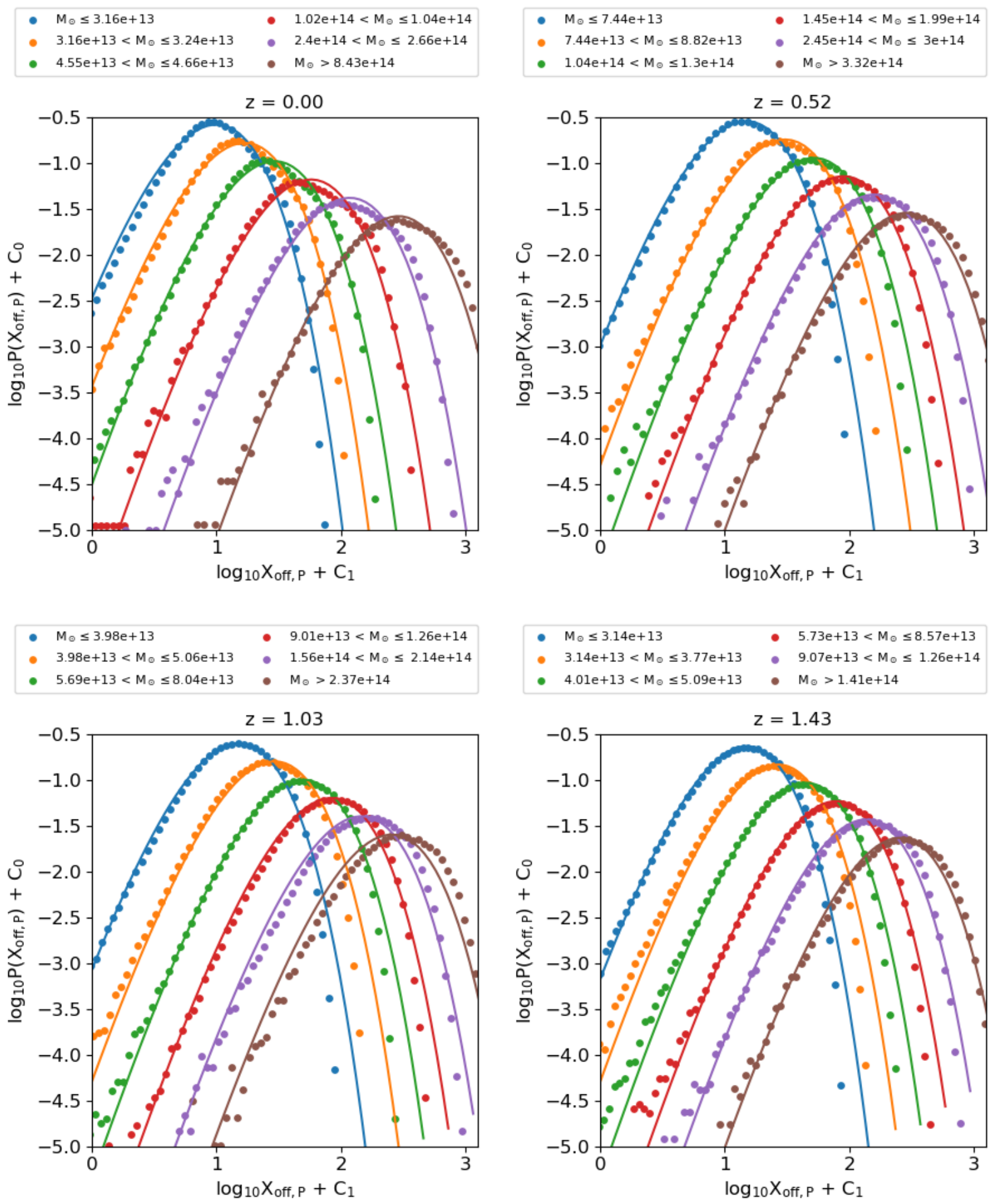

Fig. B.2. Probability density function of $X_{\text {off }, P}$ (Eq. (B.2)). Each panel shows the distribution at a specific redshift. Each set is divided in mass slices, identified by color. Scatter points indicate the data, while straight lines represent the modified Schechter model (14). For clarity, each line and its fit are shifted by 0.2 dex along both axes. This means that the coefficient $C_{0}$ assumes values $(+0.6,+0.4,+0.2,0.0,-0.2,-0.4)$, while $C_{1}$ is $(-0.6,-0.4,-0.2,0.0,+0.2,+0.4)$. The red line is not shifted, and thus is the one with the correct normalization. 
R. Seppi et al.: The mass function dependence on the dynamical state of dark matter haloes
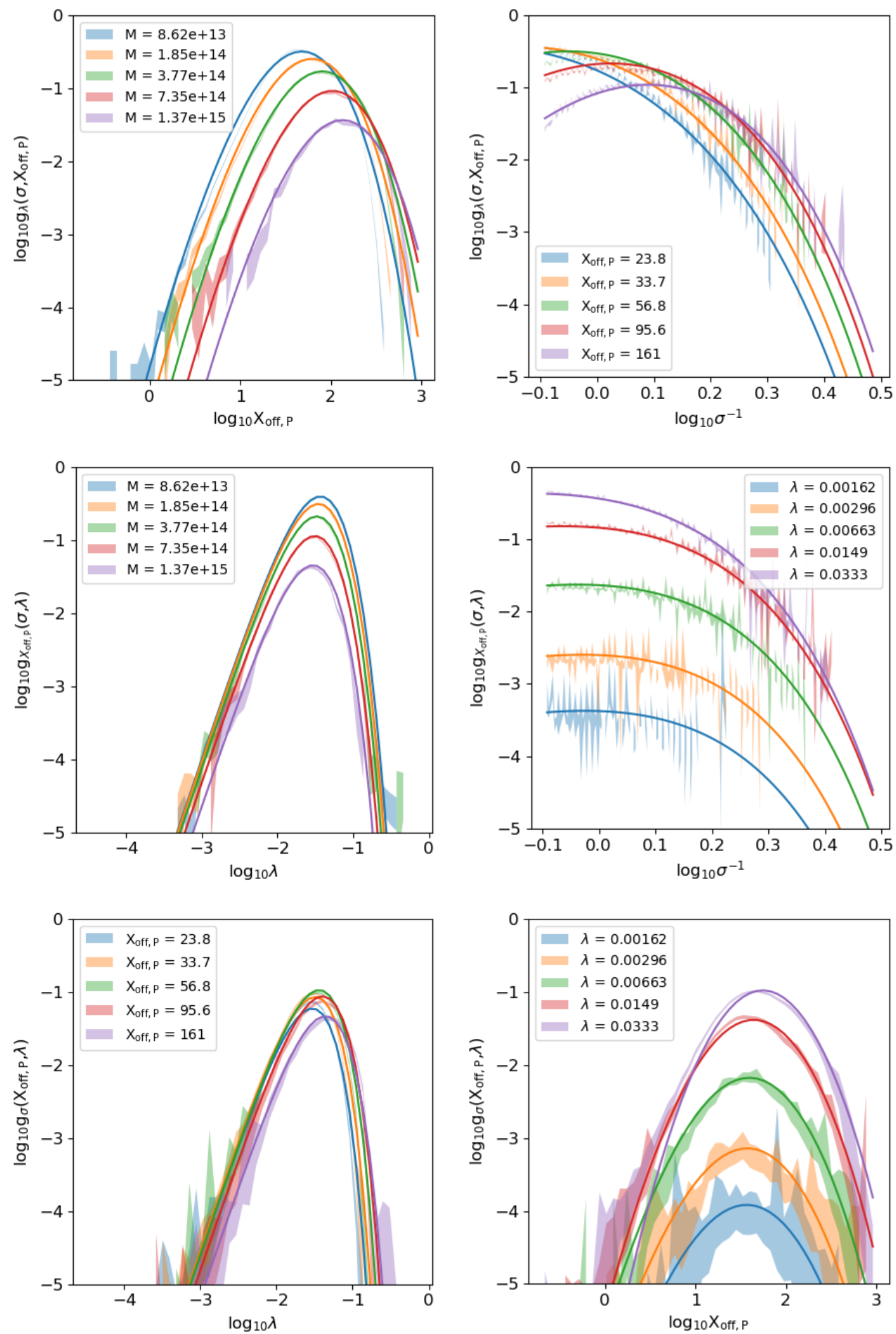

Fig. B.3. Single integration of the 3D model. In each panel straight lines indicate the best-fit model, while shaded areas represent the data with $1 \sigma$ uncertainties. Top left panel: $g_{\lambda}\left(\sigma, X_{\mathrm{off}, P}\right)$ as a function of $X_{\mathrm{off}, P}$ in different mass slices. Top right panel: $g_{\lambda}\left(\sigma, X_{\mathrm{off}, P}\right)$ as a function of $\sigma$ in different $X_{\mathrm{off}, P}$ slices. Middle left panel: $g_{X_{\mathrm{off}}, P}(\sigma, \lambda)$ as a function of $\lambda$ in different mass slices. Middle right panel: $g_{X_{\mathrm{off}}, P}(\sigma, \lambda)$ as a function of $\sigma$ in different $\lambda$ slices. Bottom left panel: $g_{\sigma}\left(X_{\mathrm{off}, P}, \lambda\right)$ as a function of $\lambda$ in different $X_{\mathrm{off}, P}$ slices. Bottom right panel: $g_{\sigma}\left(X_{\mathrm{off}, P}, \lambda\right)$ as a function of $X_{\mathrm{off}, P}$ in different $\lambda$ slices. 

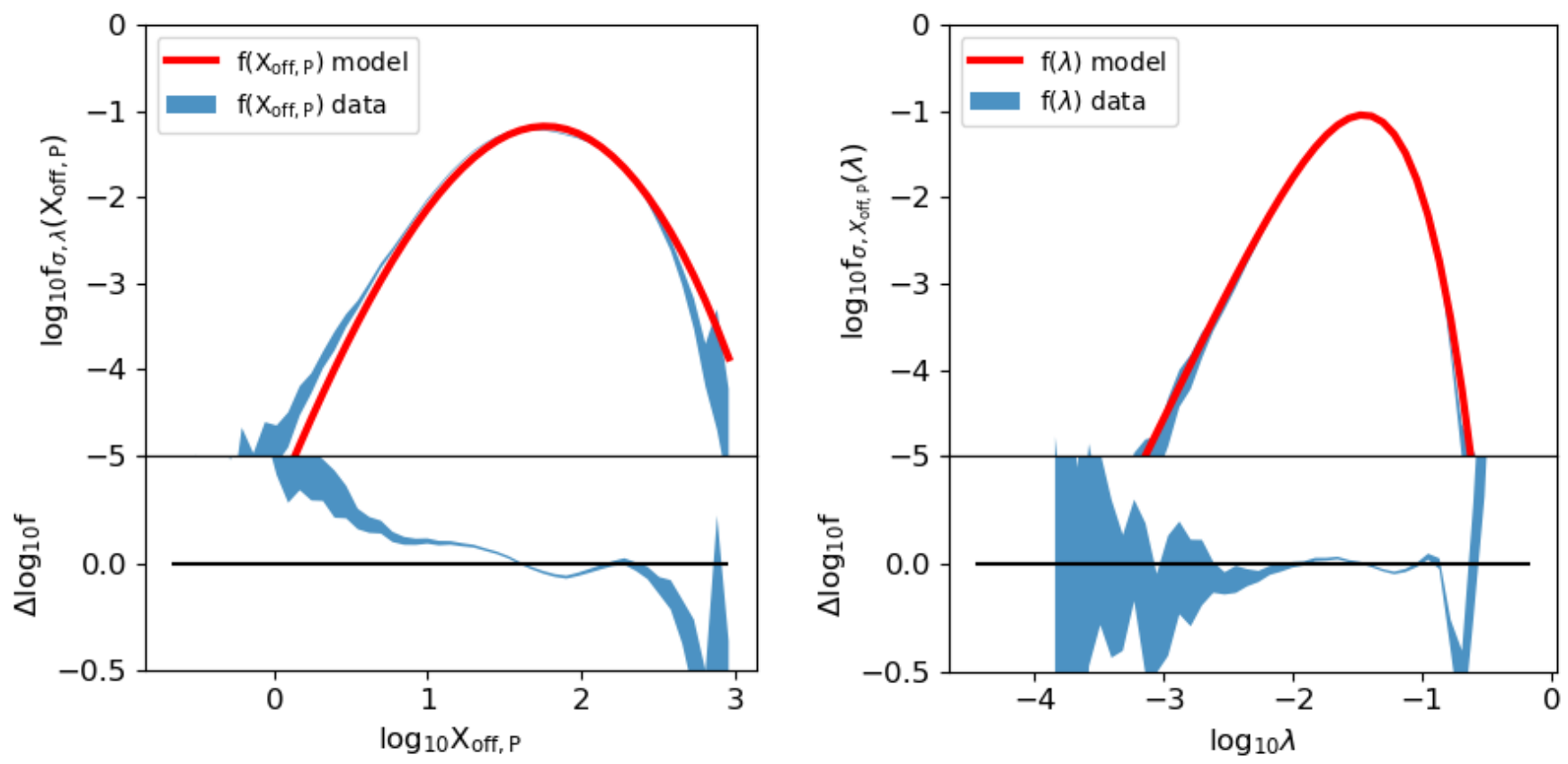

Fig. B.4. Comparison of $f\left(X_{\mathrm{off}, P}\right)$ and $f(\lambda)$ between data and model. Top panels: solid red lines indicate the integral on the best-fit model, while the shaded blue areas represent the integral on the $3 \mathrm{D} h\left(\sigma, X_{\mathrm{off}, P}, \lambda\right)$ data with $1 \sigma$ uncertainties. Each bottom panel shows the residual trend with $\sigma$ error; the straight black line represents the perfect match between data and model, with null residual. Top left panel: $f\left(X_{\mathrm{off}, P}\right)$ as a function of $X_{\text {off }, P}$. Bottom left panel: residual between $f\left(X_{\text {off, }, P}\right)$ data and model in logarithmic scale. Top right panel: $f(\lambda)$ as a function of $\lambda$. Bottom right panel: residual between $f(\lambda)$ data and model in logarithmic scale.

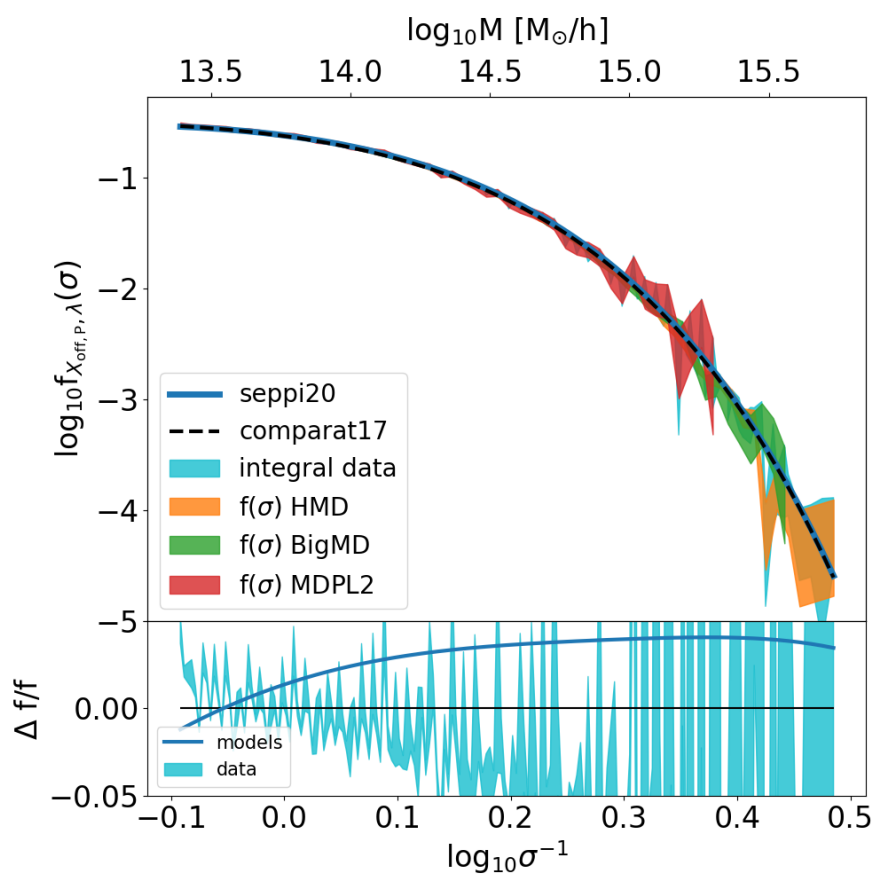

Fig. B.5. Comparison of multiplicty functions. Top panel: three shaded regions show the $1 \sigma$ contours of $f(\sigma)$ data directly computed on different simulations (orange for HMD, green for BIGMD, red for MDPL2); the light blue shaded region is the $1 \sigma$ contour of the $2 \mathrm{D}$ integral computed on the concatenated sample containing all three simulations; the dashed pink line indicates the mass function from Comparat et al. (2017), while the blue solid line is the $f(\sigma)$ we recover integrating our model along $X_{\mathrm{off}, P}$ and $\lambda$. Bottom panel: blue thick line is the fractional difference between our $f(\sigma)$ and the Comparat et al. (2017) value. The light blue shaded area represent the $1 \sigma$ contours of the residual between the integrated data and our best-fit model; the black horizontal line indicates the perfect match with null residual. 
R. Seppi et al.: The mass function dependence on the dynamical state of dark matter haloes



Fig. B.6. Redshift evolution of the best-fit parameters for $h\left(\sigma, X_{\mathrm{off}, P}, \lambda\right)$. Each panel shows a single parameter. The values at $z=0$ are reported in Table B.2. The slopes of the redshift trends are given in Table B.3.

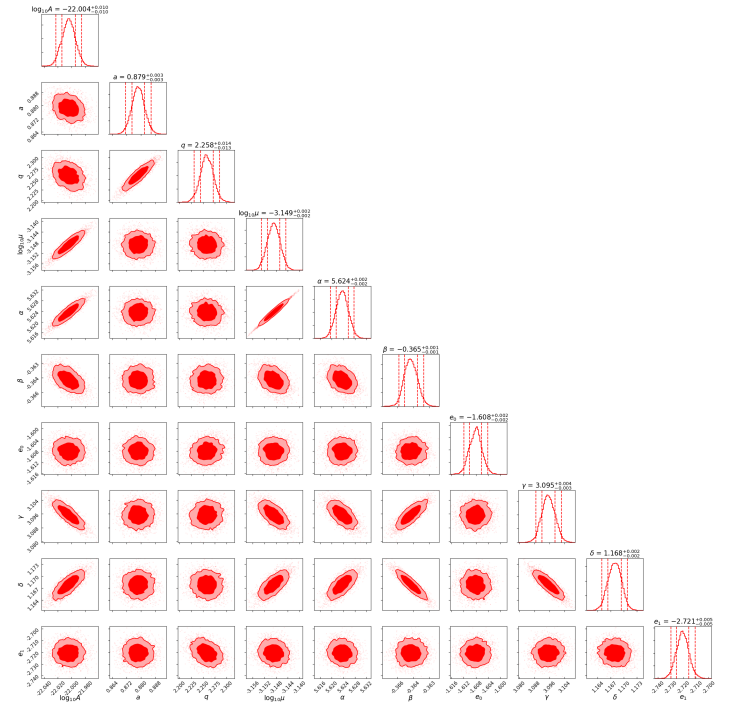

Fig. B.7. Marginalized posterior distributions of the $h\left(\sigma, X_{\mathrm{off}, P}, \lambda\right)$ bestfit parameters at redshift 0 . The 0.68 and 0.95 confidence levels of the posteriors are shown as filled $2 \mathrm{D}$ contours. The 2.5 th, 16 th, 84 th, and 97.5th percentiles of the one-dimensional posterior distributions are indicated by the vertical lines on the diagonal plots.

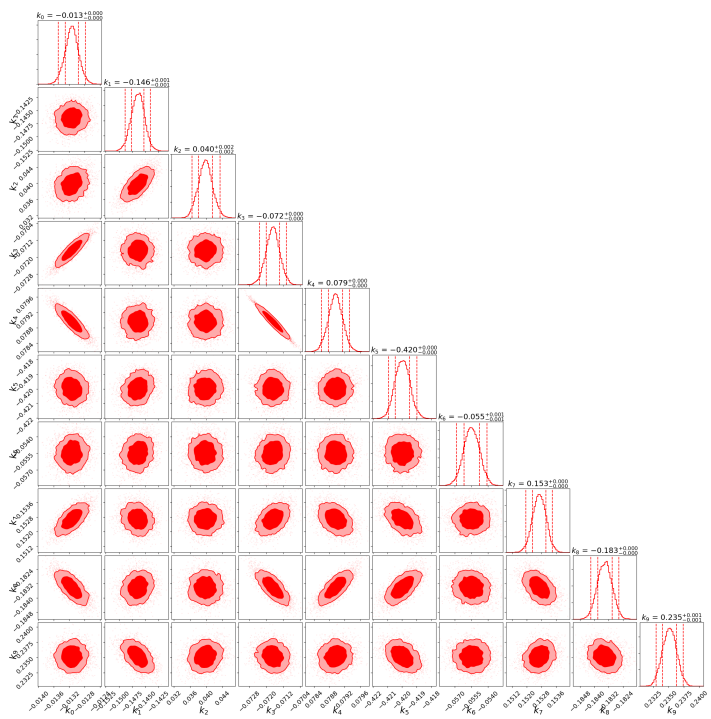

Fig. B.8. Marginalized posterior distributions of the best-fit parameters describing the redshift evolution of $h\left(\sigma, X_{\mathrm{off}, P}, \lambda\right)$. The 0.68 and 0.95 confidence levels of the posteriors are shown as filled 2D contours. The 2.5 th, 16th, 84th, and 97.5th percentiles of the one-dimensional posterior distributions are indicated by the vertical lines on the diagonal plots. 